\title{
PolarCam micropolarizer cameras characterization and usage
}

\author{
A. Liberatore, S. Fineschi, G. Capobianco, G. Massone, L. \\ Zangrilli, et al.
}

A. Liberatore, S. Fineschi, G. Capobianco, G. Massone, L. Zangrilli, G. Nicolini, R. Susino, "PolarCam micropolarizer cameras characterization and usage," Proc. SPIE 11852, International Conference on Space Optics ICSO 2020, 118520W (11 June 2021); doi: 10.1117/12.2599180 SPIE. Event: International Conference on Space Optics — ICSO 2021, 2021, Online 


\section{International Conference on Space Optics-ICSO 2020}

Virtual Conference

30 March-2 April 2021

Edited by Bruno Cugny, Zoran Sodnik, and Nikos Karafolas
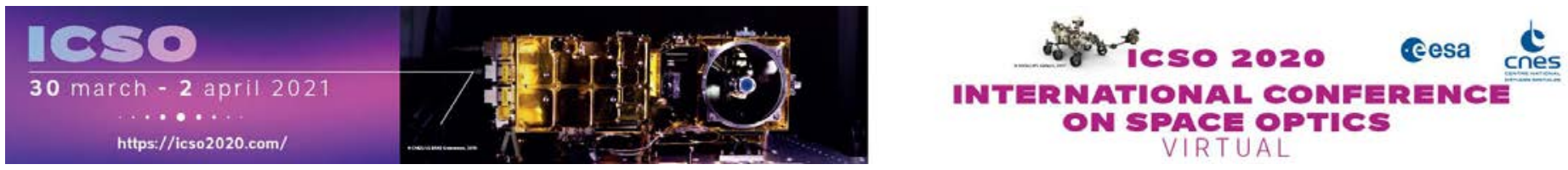

\section{PolarCam micropolarizer cameras characterization and usage}

\section{Cesa isopueatans lecnes}

International Conference on Space Optics - ICSO 2020, edited by Bruno Cugny, Zoran Sodnik,

Nikos Karafolas, Proc. of SPIE Vol. 11852, 118520W · (C) 2021 ESA and CNES

CCC code: $0277-786 \mathrm{X} / 21 / \$ 21 \cdot$ doi: $10.1117 / 12.2599180$ 


\title{
PolarCam micropolarizer cameras characterization and usage
}

\author{
A. Liberatore ${ }^{a}$, S. Fineschi ${ }^{a}$, G. Capobianco ${ }^{a}$, G. Massone ${ }^{a}$, L. Zangrilli ${ }^{a}$, G. Nicolini ${ }^{a}$, and R. \\ Susino $^{\mathrm{a}}$ \\ ${ }^{a}$ INAF - Astrophysical Observatory of Turin; Via Osservatorio 20, 10025 Pino Torinese, Italy
}

\begin{abstract}
Several experiments need to acquire images with different linear polarisation. Acquisition of successive images through a rotating polarising elements give the possibility to achieve this goal. However, the subsequent acquisition of these images implies a temporal modulation and a rotation mechanism (with a consequent consumption of space, power, etc...). The PolarCam ${ }^{\circledR}$, a camera where an array of linear micropolarizers with orientation are matching the sensor's pixels, tries to solve these problems. In the present manuscript the features of this camera and the main aspects of its calibration are shown. Finally, an example of its application is presented. In particular, our application of this camera is for the Antarctica solar Coronagraph -AntarctiCor- for the "Extreme Solar Coronagraphy Antarctic Program Experiment" -ESCAPE- that used this technology for a ground-based polarimetric study of the solar K-corona from the Italian-French Concordia Base (Dome C - Antarctica).
\end{abstract}

Keywords: PolarCam, micropolarizer, polarisation, camera characterisation, coronagraphy, Antarctica.

\section{INTRODUCTION}

In more and more fields, from microscopy to space science, it is required to acquire images with different polarisation. Commonly, the acquisition of polarised images is performed through the use of a linear polarizer placed in front of the instrument sensor. By rotating this linear polarizer, the temporal resolution worsens. The temporal resolution can be greatly improved by acquiring all the necessary polarisation at the same time, using spatial modulation instead of temporal. The polarisation cameras do that. There are many polarisation cameras in the market. In particular, in this manuscript, we consider the PolarCam ${ }^{\odot}$; the polarisation camera that we used for the "ESCAPE Project" in Antarctica (Sec. 4.1). The PolarCam is manufactured from 4-D Technology Corporation, Arizona, United States. This camera capture images of multiple polarised angles $\left(0^{\circ}, 45^{\circ}, 90^{\circ}\right.$ and $135^{\circ}$ ) at the same time thanks to an array of linear micropolarizers on the camera sensor (different orientation matching different sensor's pixels - see Fig. 1). Furthermore, the presence of this micropolarizer grid eliminate the need of a linear polarizer and the associated rotation mechanisms. This implies less weight, less space needed, less power consumption and a general decrease of possible instruments malfunctions. In this manuscript, after a brief summary about the polarimetric measurements (Subec. 1.1), a more detailed description of this camera is given in Section 2. After that, a first characterisation of the PolarCam is shown in Section 3. Finally, before the Conclusion 5, an example of its application in Solar physics is discussed in Section 4 before an introduction to the physics of the solar corona in which the need for polarimetric acquisitions is highlighted.

\subsection{Introduction to polarimetric measurements}

This Subsection is meant to provide to the reader an introduction to polarimetry by getting in all the essential elements necessary for the rest of the manuscript. Anyhow, there are numerous sources of reference for a detailed introduction to polarimetry; more information about polarimetry and its application can be found, for example, in Ref. 2.

Further author information: (Send correspondence to Alessandro Liberatore or Silvano Fineschi)

Alessandro Liberatore: E-mail: alessandro.liberatore@inaf.it, Telephone: (+39) 3473689597

Silvano Fineschi: E-mail: silvano.fineschi@inaf.it, Telephone: (+39) 0118101919 


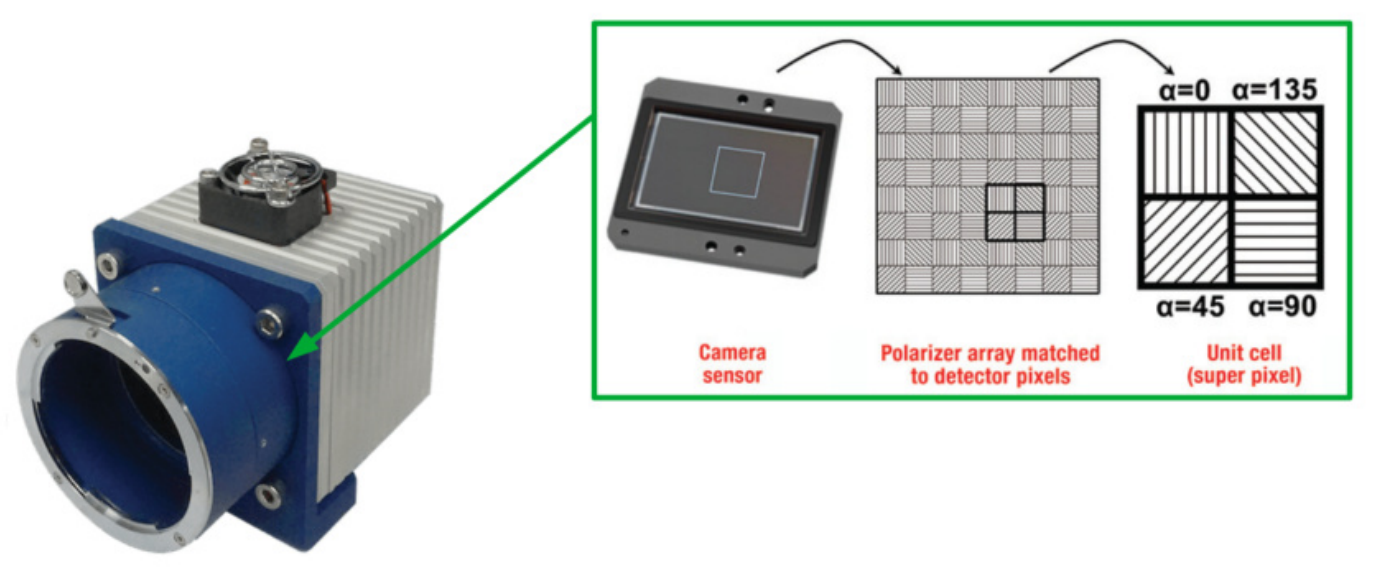

Figure 1. PolarCam and its sensor with the pixelated micropolarizer array. ${ }^{1}$

In general, a complete polarimetric study of the light incident to the detector sensor requires the evaluation of the Stokes vector $\mathbf{S}$. The Stokes vector elements are defined as follow:

$$
\mathbf{S}=\left[\begin{array}{c}
S_{0} \\
S_{1} \\
S_{2} \\
S_{3}
\end{array}\right]=\left[\begin{array}{c}
I_{0}+I_{90} \\
I_{0}-I_{90} \\
I_{45}-I_{135} \\
I_{L H C}-I_{R H C}
\end{array}\right]
$$

where $I_{0}, I_{90}, I_{45}, I_{135}$, are the intensities of the linear polarisation components at $0,90,45,135$ degrees and $I_{L H C}, I_{R H C}$ are the left hand and right hand circular polarisation intensities respectively. Then, results to be $S_{0}^{2} \geq S_{1}^{2}+S_{2}^{2}+S_{3}^{2}$. Commonly, the Stokes elements $S_{0}, S_{1}, S_{2}, S_{3}$ are also reported in literature with the notation $I, Q, U, V$ respectively. In particular, the linearly polarised light requires the measurement of the I, Q, $\mathrm{U}$ quantities to be fully characterised. The $\mathrm{V}$ parameters is associated to the circular polarisation and it is not relevant to the work being done in this manuscript. There are several equations that relate all these quantities. In particular:

$$
\begin{aligned}
& Q=I p \cos 2 \theta \\
& U=I p \sin 2 \theta
\end{aligned}
$$

where $p$ is the Degree of Linear Polarisation (DoLP) and $\theta$ is the Angle of Linear Polarisation (AoLP). Then, this two quantities can be expressed, relatively to the detector axis as:

$$
\begin{aligned}
& \operatorname{DoLP}[\%]=\frac{\sqrt{Q^{2}+U^{2}}}{I} \cdot 100 \\
& \operatorname{AoLP}[\mathrm{rad}]=\frac{1}{2} \arctan \left(\frac{U}{Q}\right)
\end{aligned}
$$

Another quantity often used in polarimetric studies is the polarimetric brightness defined as:

$$
p B=\sqrt{Q^{2}+U^{2}}
$$

There are other possible representations that will not be discussed in this paper. Anyhow, all these intrinsic properties of the radiation should not be confused with the properties of the polarising elements (see Subsec. 3.4).

Finally, it is good to introduce the concept of demodulation tensor $\mathbf{X}^{\dagger}$. As we will see in the next Sections, part of the PolarCam characterisation aims to achieve the camera $\mathbf{X}^{\dagger}$. This tensor (obtained from a modulation 
matrix $\mathbf{X}$ associated with each pixel of the acquired polarimetric images) allow us to polarimetrically characterise the incoming light founding the associated Stokes vector $\mathrm{S}^{*}$ as shown in Eq. 7 (where $m$ is the vector of the acquired images at different polarisation).

$$
\mathbf{m}=\mathbf{X S} \rightarrow \mathbf{S}=\mathbf{X}^{\dagger} \mathbf{m}
$$

According to the equations showed before, it is necessary to acquire images at 4 different polarizations and, as mentioned, the PolarCam, with a single shot, can give us images at $\theta=\left(0^{\circ}, 45^{\circ}, 90^{\circ}, 135^{\circ}\right)$. Then, in our case, we have $m=\left(m_{0}, m_{45}, m_{90}, m_{135}\right)$.

From the Mueller matrix ${ }^{2}$ it is possible to obtain a theoretical modulation matrix $\mathbf{X}$. In particular, considering the angles $\theta_{i}=\left(0^{\circ}, 45^{\circ}, 90^{\circ}, 135^{\circ}\right)$, it results to be equal to:

$$
\mathbf{X}=\frac{1}{2}\left(\begin{array}{ccc}
1 & \cos 2 \theta_{1} & \sin 2 \theta_{1} \\
1 & \cos 2 \theta_{2} & \sin 2 \theta_{2} \\
1 & \cos 2 \theta_{3} & \sin 2 \theta_{3} \\
1 & \cos 2 \theta_{4} & \sin 2 \theta_{4}
\end{array}\right)=\frac{1}{2}\left(\begin{array}{ccc}
1 & 1 & 0 \\
1 & 0 & 1 \\
1 & -1 & 0 \\
1 & 0 & -1
\end{array}\right)
$$

Now we can retrieve the theoretical demodulation matrix $\mathbf{X}^{\dagger}$ as Moore-Penrose inverse of the theoretical modulation matrix $\mathbf{X}$. We obtain:

$$
\mathbf{X}^{\dagger}=\frac{1}{2}\left(\begin{array}{cccc}
1 & 1 & 1 & 1 \\
2 & 0 & -2 & 0 \\
0 & 2 & 0 & -2
\end{array}\right)=\left(\begin{array}{cccc}
0.5 & 0.5 & 0.5 & 0.5 \\
1 & 0 & -1 & 0 \\
0 & 1 & 0 & -1
\end{array}\right)
$$

Then, the theoretical Stokes parameters can be easily obtained just solving Eq. 7:

$$
\left(\begin{array}{l}
I \\
Q \\
U
\end{array}\right)=\left(\begin{array}{cccc}
0.5 & 0.5 & 0.5 & 0.5 \\
1 & 0 & -1 & 0 \\
0 & 1 & 0 & -1
\end{array}\right)\left(\begin{array}{l}
m_{1} \\
m_{2} \\
m_{3} \\
m_{4}
\end{array}\right)=\mathbf{X}^{\dagger}\left(\begin{array}{l}
m_{1} \\
m_{2} \\
m_{3} \\
m_{4}
\end{array}\right)
$$

Performing this study pixel by pixel we obtain the full theoretical demodulation tensor $X^{\dagger}$ instead of a single matrix. The results in the next Section were obtained by using both the theoretical demodulation tensor and the one we got during the PolarCam calibration campaign (Sec. 3.4).

\section{POLARCAM DESCRIPTION}

Before to going into the PolarCam description it is good to remember that the general camera features change in function of the camera model (G1, G2, U2, U4). ${ }^{1}$ In particular, we considered U4 model and, from now on, even if not explicitly stated, all information and results obtained are based on this model.

The PolarCam consists of a camera whose sensor has a (micro)polarizer array upon a charge-coupled device (CCD). This array consists of repeated "super-pixels" composed of a fixed pattern of four pixels (Fig. 1). The linear polarizers pattern in the super-pixel is composed of four discrete polarisations at angles $0^{\circ}, 45^{\circ}, 90^{\circ}$ and $135^{\circ}$. The upper left pixel of the camera is a 0-phase (vertical polarisation) state.

This camera has a monochrome CCD of size $1950 \times 1950$ pixels. Each pixel has dimensions $7.4 \mu \mathrm{m} \times 7.4 \mu \mathrm{m}$ with a 12 bit depth. The maximum camera frame-rate is equal to $14 \mathrm{fps}$. The nominal main features of PolarCam are summarised in Tab. 1. Furthermore, the camera has a control software and a Software Development Kit (SDK) that assists the user to access information from PolarCam for analysis and further manipulation. From the software is possible, for example, change the exposure time (minimum $T_{\exp }=0.02 \mu \mathrm{s}$ ). Moreover, being the PolarCam detector managed by two different ADCs, it is possible, through the software, set a particular gain value for each one. For this reason, when we will talk about analog gain (AG), we will always report two values.

${ }^{*}$ Being the $S_{3}(\equiv V)$ parameter associated with circular polarisation (and considering that the camera is sensible just to the linear one) we are only interested in the first three Stokes parameters $(\equiv[I, Q, U])$. 
Table 1. PolarCam U4 model main features. ${ }^{1}$

\begin{tabular}{r|l}
\hline Sensor Type & Interline transfer CCD ON \\
& Semiconductor KAI04070 \\
Micropolarizers orientation & $0^{\circ}, 45^{\circ}, 90^{\circ}, 135^{\circ}$ \\
Pixel Size & $7.4 \mu \mathrm{m} \times 7.4 \mu \mathrm{m}$ \\
Usable Pixels & $1950 \times 1950,3.8 \mathrm{MP}$ \\
Frame Rate & $14 \mathrm{fps}$ \\
Saturation Capacity & $44 \mathrm{ke}^{-}$ \\
Dark Noise & $3 \mathrm{e}^{-} / \mathrm{s}$ \\
Quantum Efficiency & $76 \% @ 470 \mathrm{~nm}$ \\
Physical Envelope & $60 \times 60 \times 95 \mathrm{~mm}$ \\
Weight & $210 \mathrm{~g}$ \\
Power Requirement & $8 \mathrm{~W}, 12 \mathrm{VDC}$ \\
Interface & USB 3.0 \\
Lens Mounting Type & F-Mount \\
\hline
\end{tabular}

\subsection{Raw image demosaicing}

To obtain the images at different polarisation $\left(0^{\circ}, 45^{\circ}, 90^{\circ}, 135^{\circ}\right)$ from the original raw, a demosaic process is required. In Fig. 2 an example of a possible way to perform this process is shown. In particular, once a certain polarization angle has been chosen $\left(e . g .0^{\circ}\right)$, the method used during our analysis was to consider the 3 remaining pixels, for each super-pixel, as the average between the considered pixel and the values that the pixels with the chosen polarisation have in each adjacent super-pixel (i.e. "Output 2" in Fig. 2). The same procedure can be applied to get the images with the other polarisation.

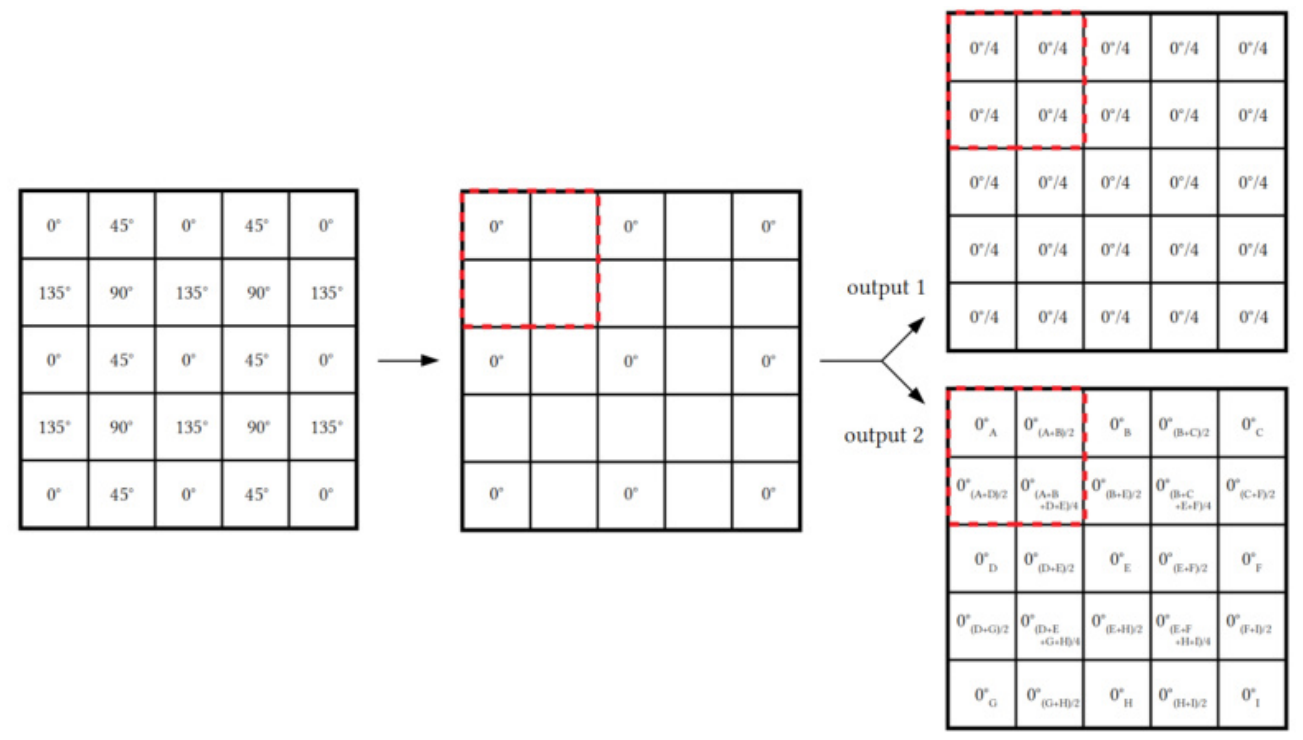

Figure 2. Demosaic example to obtain a polarised image from the original raw. In this particular example it is shown how to obtain the image with a polarisation angle of 0 degrees $\left(I_{0}\right)$. Output 1: each super-pixel value was obtained from the pixel value with the micropolarizer at 0 degrees. Output 2: each super-pixel value was obtained considering also the pixel values with the micropolarizer at 0 degrees in the adjacent super-pixels. 


\section{POLARCAM CHARACTERISATION}

All the following PolarCam characterisations were performed in the Astrophysical Observatory of Turin (OATo) clean room - ISO7 or in the OATo Space Optics Calibration Chamber (SPOCC) placed at the Aerospace Logistics Technology Engineering Company (ALTEC) in Turin, Italy.

\subsection{Detector resolution}

To evaluate the detector resolution we used the PolarCam looking at a resolution target USAF-1951 ${ }^{3}$ illuminated by a white led source with a light diffuser, powered at $3.2 \mathrm{~V}$ (Fig. 3). A collimator was placed between the detector and the resolution target. The collimator aperture is $\approx 50 \mathrm{~mm}$ with a focal length of $\approx 300 \mathrm{~mm}$.

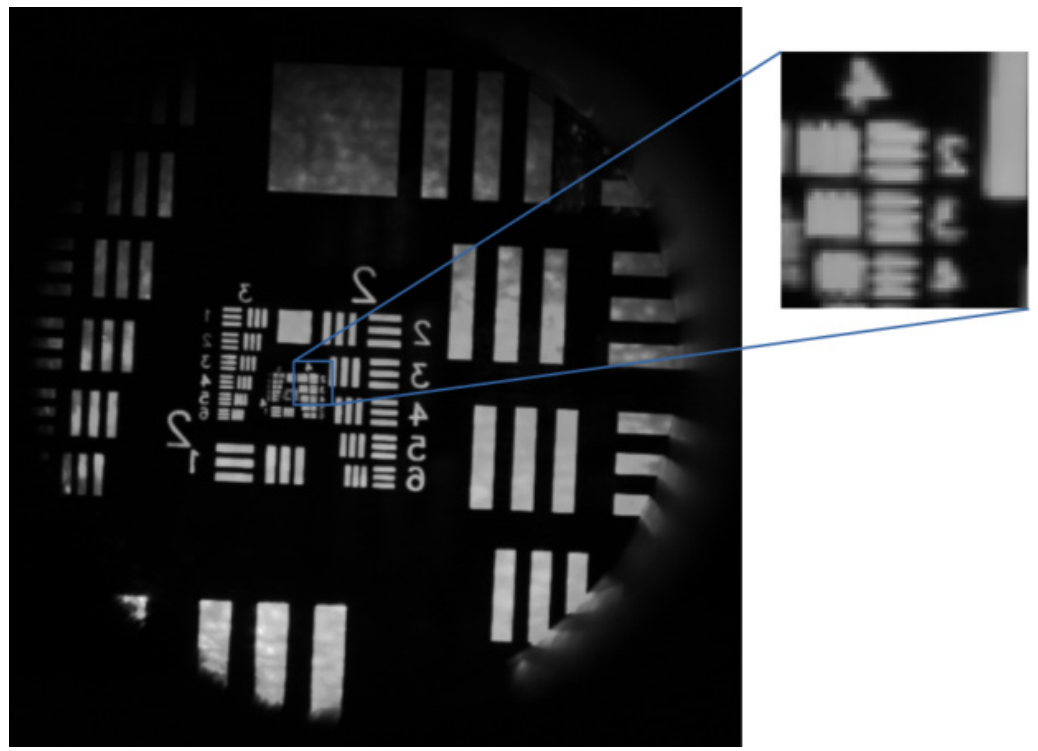

Figure 3. An image of a resolution target as seen by the PolarCam (i.e. the AntarctiCor detector) with gain $=1$ and $T_{e x p}=71.86 \mathrm{~ms}$. To avoid values conditioned by the polarisation, this image is obtained as the sum of the intensities of the linear polarisation components at 0 and 90 degrees (i.e. the first element of the Stokes vector).

Thanks to a Modulation Transfer Function, it is possible to evaluate the resolution through the Rayleigh criterion (Figure 4). In particular, when the modulation is equal to 0.2 we obtain that the frequency is almost 22 [linepairs $/ \mathrm{mm}$ ] (i.e. $\approx 22 \mu \mathrm{m}$ ) that corresponds to the group 4 element 4 of the resolution target (Table 2 ).

Table 2. Line pairs per millimetre for each group element of the resolution target. ${ }^{3}$

Element Number

\begin{tabular}{|c|cccccccc|}
\hline & $\mathbf{0}$ & $\mathbf{1}$ & $\mathbf{2}$ & $\mathbf{3}$ & $\mathbf{4}$ & $\mathbf{5}$ & $\mathbf{6}$ & $\mathbf{7}$ \\
\hline $\mathbf{1}$ & 1.00 & 2.00 & 4.00 & 8.00 & 16.00 & 32.00 & 64.00 & 128.00 \\
\hline $\mathbf{2}$ & 1.12 & 2.24 & 4.49 & 8.89 & 17.96 & 35.92 & 71.84 & 143.70 \\
\hline $\mathbf{3}$ & 1.26 & 2.52 & 5.04 & 10.08 & 20.16 & 40.32 & 80.63 & 161.30 \\
\hline $\mathbf{4}$ & 1.41 & 2.83 & 5.66 & 11.31 & 22.63 & 45.25 & 90.51 & 181.00 \\
\hline $\mathbf{5}$ & 1.59 & 3.17 & 6.35 & 12.70 & 25.40 & 50.80 & 101.60 & 203.20 \\
\hline $\mathbf{6}$ & 1.78 & 3.56 & 7.13 & 14.25 & 28.51 & 57.02 & 114.00 & 228.10 \\
\hline
\end{tabular}




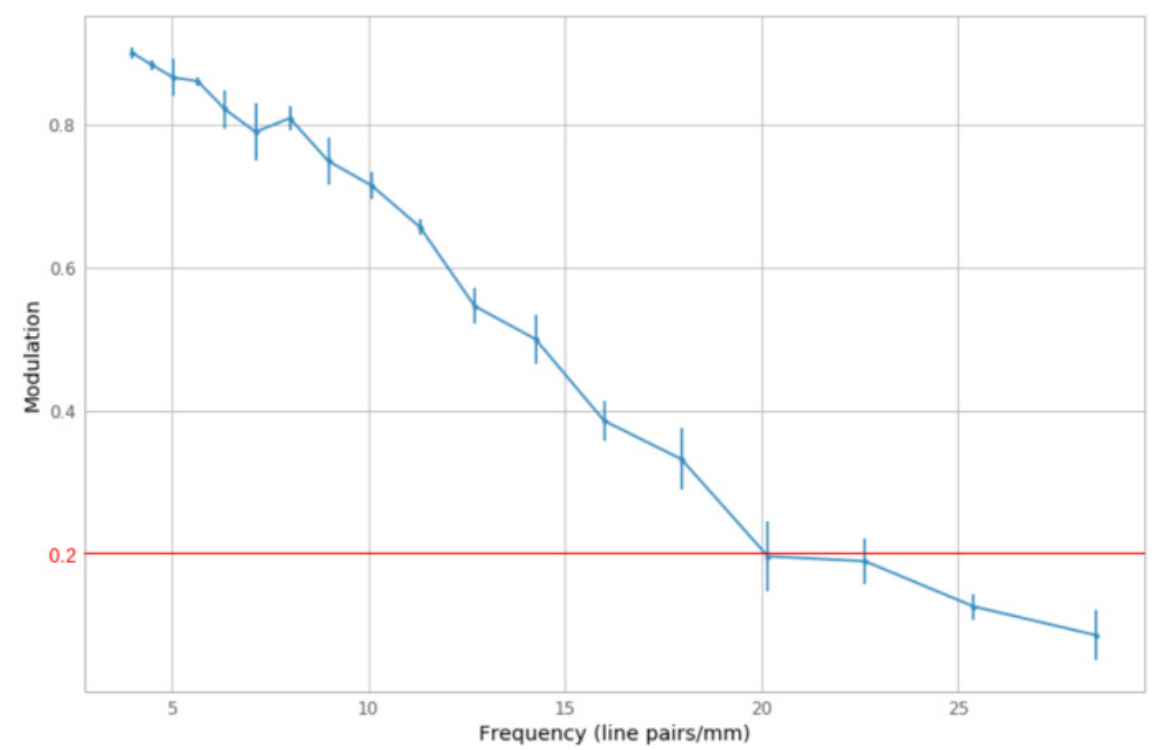

Figure 4. PolarCam Modulation Transfer Function (MTF).

\subsection{Detector linearity}

Changing the exposure time it was possible to check the PolarCam linear response (Fig. 5). During these acquisitions we set an analog gain of $\mathrm{AG}=(44,44)$ and a digital gain $\mathrm{DG}=1$ (it is possible to set a digital gain from a minimum of $\mathrm{DG}=1$ until a maximum $\mathrm{DG}=16$, engineering units). In particular, the camera linear response was evaluated by averaging over the entire frame and by separating the contributions given by the pixels with different orientation of the micropolarizers. It is possible to check also the average dark for different exposure time and different digital gain. What we obtain is shown in Fig. 6. As expected, the average dark values increase for higher DG. Moreover, it is almost constant in the considered exposure time range.

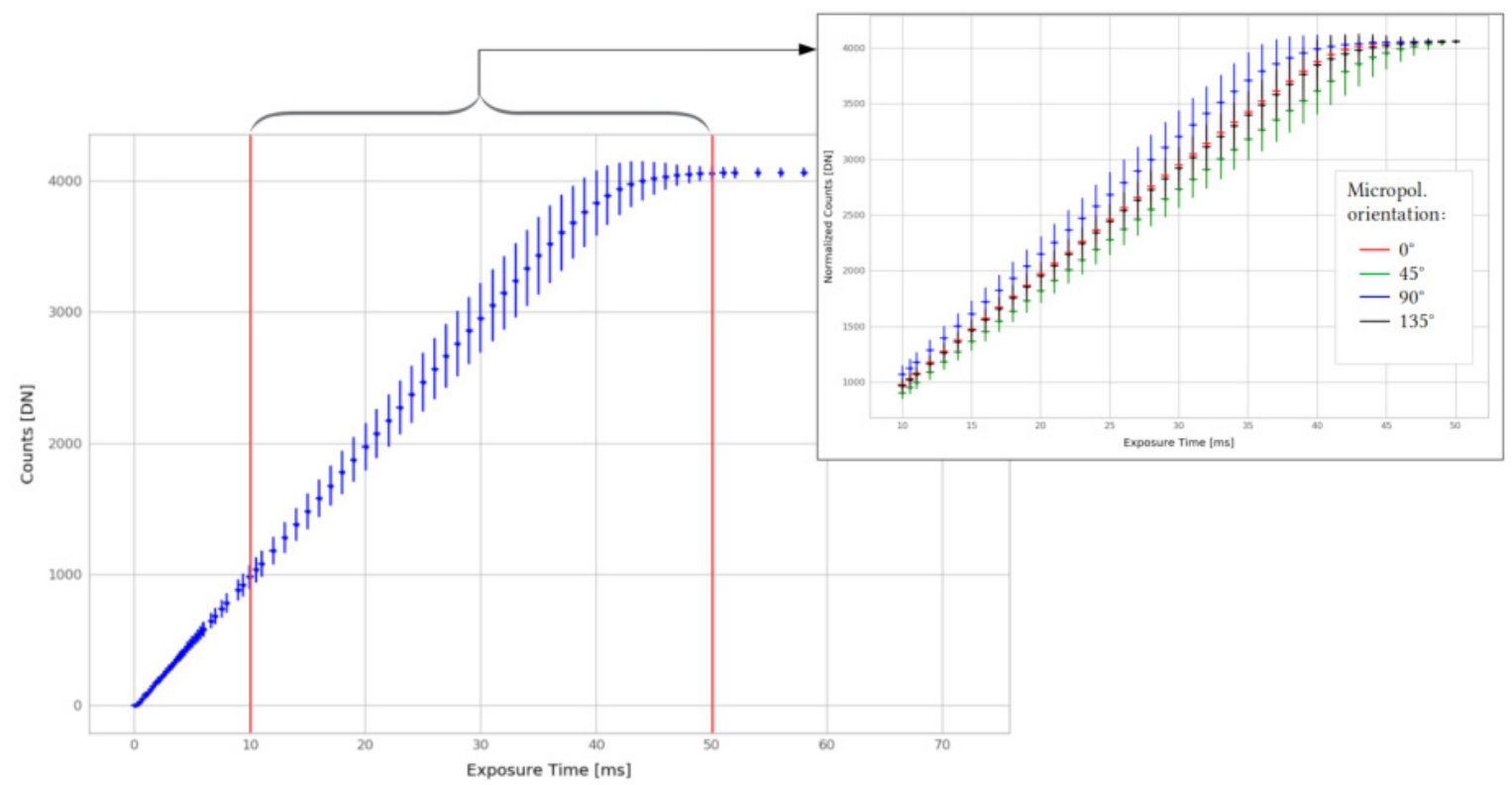

Figure 5. PolarCam linear response averaging over the whole frame and considering the four different micropolarizer array orientation (in the grey box). 


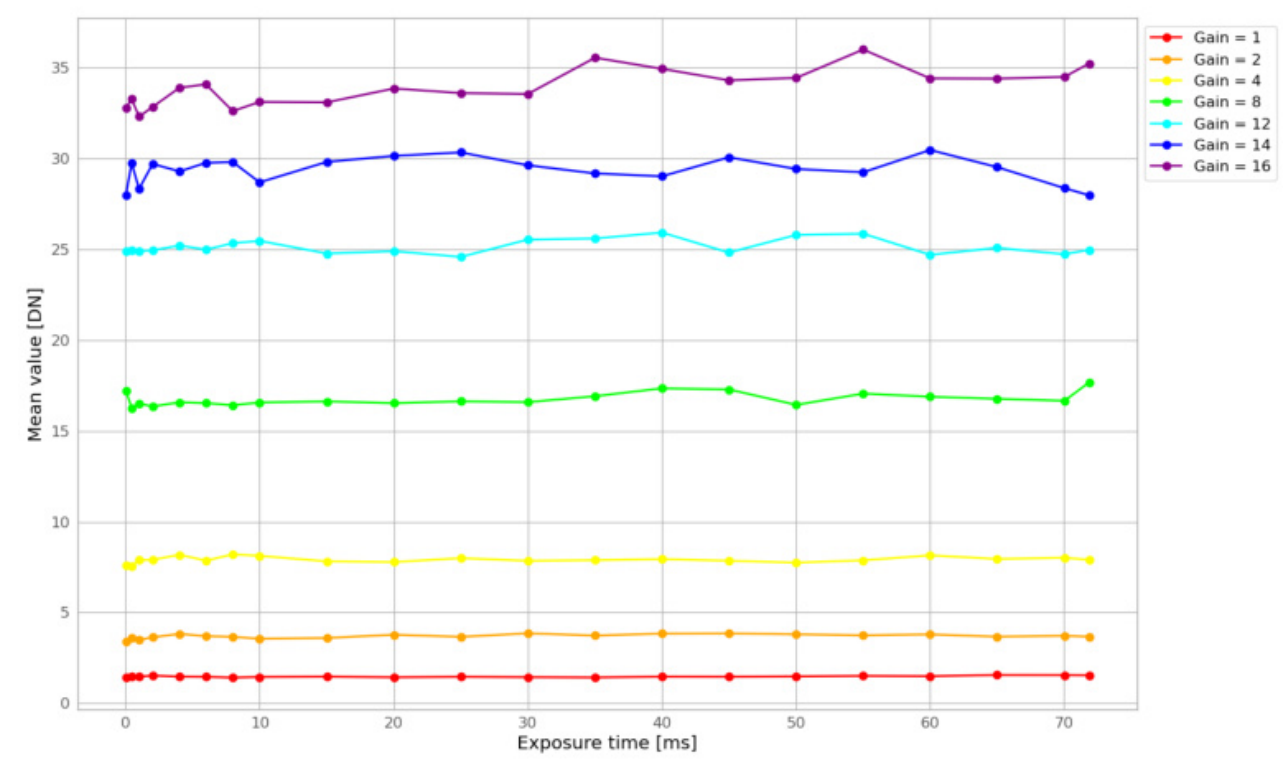

Figure 6. PolarCam dark measurement for different digital gain and fixed analog gain.

\subsection{Angle and Degree of Linear Polarisation}

As introduced in Sec. 1, with a single shot, we can evaluate the Stokes I, Q and U parameters using the theoretical demodulation tensor (as explained in Subsec. 2.1) and then calculate the DoLP and AoLP from them (Eqs. 4 and 5).

Considering a flat-field panel as light source (i.e., unpolarized light source), what we expect to obtain looking at the DoLP is an almost zero degree of polarisation. The measured DoLP could be interpreted as instrumental polarisation, or the polarisation introduced by the PolarCam itself and as a consequence it will not be possible to measure a polarisation lower than this one. The results are shown in Fig. 7 (right side). It is possible to see that the polarisation introduced by the camera on the left side of the frame is at the order of $\sim 4 \%$ on the Stokes $\mathrm{Q}$ and $\mathrm{U}$ parameters and as shown in the right panel, the DoLP increase up to the $\sim 10 \%$. The average of the degree of linear polarisation on the whole frame is: $(6 \pm 2) \%$.

In addition to this unpolarized flat-field measurements, we performed also the same kind of measurement by introducing a linear polarizer (pre-polarizer) between the detector and the flat-field panel, obtaining a polarimetric flat-field. By rotating the pre-polarizer, at the detector plane, the light is fully linearly polarised in the direction defined by the orientation of the acceptance axis of the pre-polarizer. Therefore, what we expect to observe is a DoLP of $\sim 100 \%$ and the AoLP having the same angle of the acceptance axis of the pre-polarizer. As an example, in Figs. 8 and 9 are shown the DoLP and the AoLP measured by the PolarCam when the pre-polarizer is oriented at the four main angles of $0^{\circ}, 45^{\circ}, 90^{\circ}$ and $135^{\circ}$. The results are summarised in Tab. 3.

Table 3. unpolarised flat-field (UFF) and polarimetric flat-field (PFF) DoLP and AoLP evaluated by the application of the theoretical demodulation tensor.

\begin{tabular}{|c|c|c|c|c|}
\hline Set up & Expected DoLP & Obtained DoLP & Expected AoLP & Obtained AoLP \\
\hline $\mathrm{UFF}$ & $0 \%$ & $(6 \pm 2) \%$ & $/$ & $/$ \\
$\mathrm{PFF}\left(0^{\circ}\right)$ & $100 \%$ & $(91 \pm 2) \%$ & $0^{\circ}$ & $\left(2^{\circ} \pm 1^{\circ}\right)$ \\
$\mathrm{PFF}\left(45^{\circ}\right)$ & $100 \%$ & $(85 \pm 2) \%$ & $45^{\circ}$ & $\left(41^{\circ} \pm 1^{\circ}\right)$ \\
$\mathrm{PFF}\left(90^{\circ}\right)$ & $100 \%$ & $(96 \pm 2) \%$ & $90^{\circ}$ & $\left(91^{\circ} \pm 1^{\circ}\right)$ \\
$\mathrm{PFF}\left(135^{\circ}\right)$ & $100 \%$ & $(87 \pm 2) \%$ & $135^{\circ}$ & $\left(128^{\circ} \pm 1^{\circ}\right)$ \\
\hline
\end{tabular}



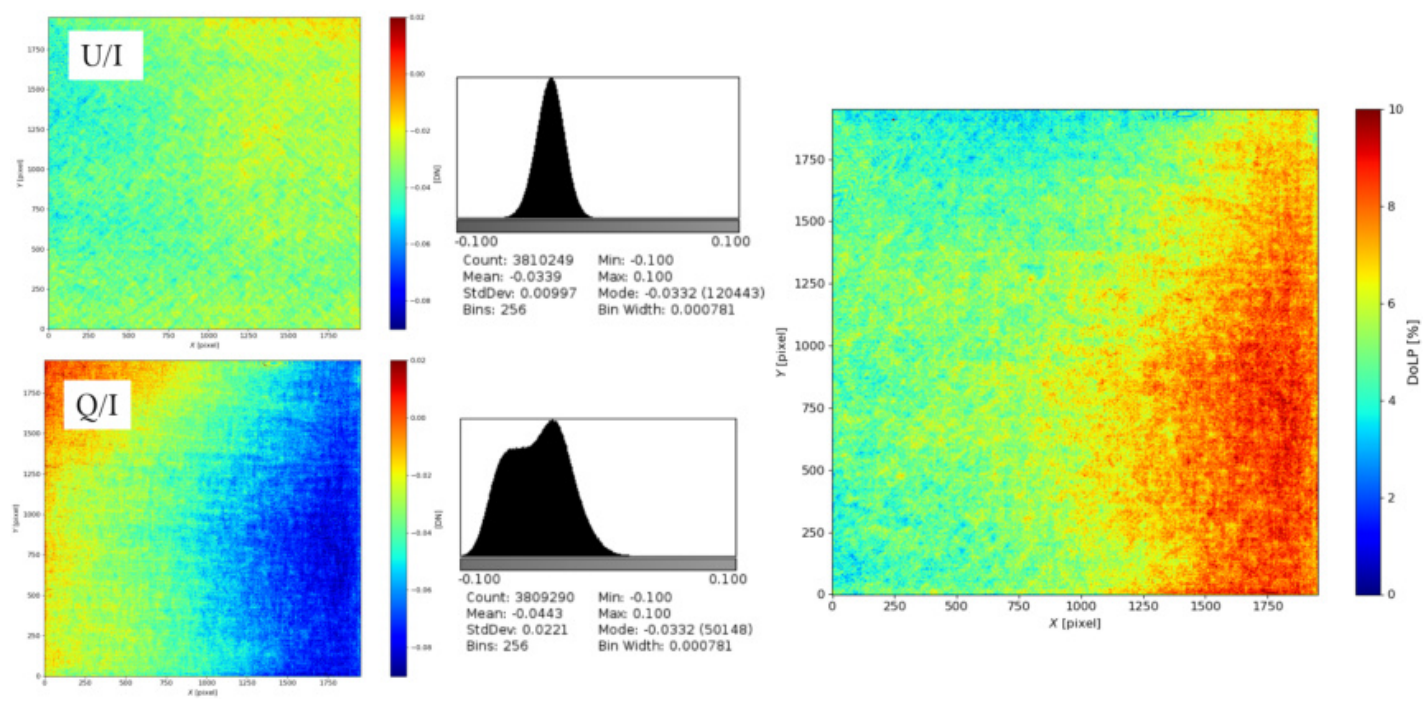

Figure 7. Test with flat-field (unpolarised light). Left side: Stokes parameters obtained with the theoretical demodulation tensor (Stokes vector $=[1, \mathrm{Q} / \mathrm{I}, \mathrm{U} / \mathrm{I}]$ ). Right side: Degree of Linear Polarisation obtained with a theoretical demodulation tensor.
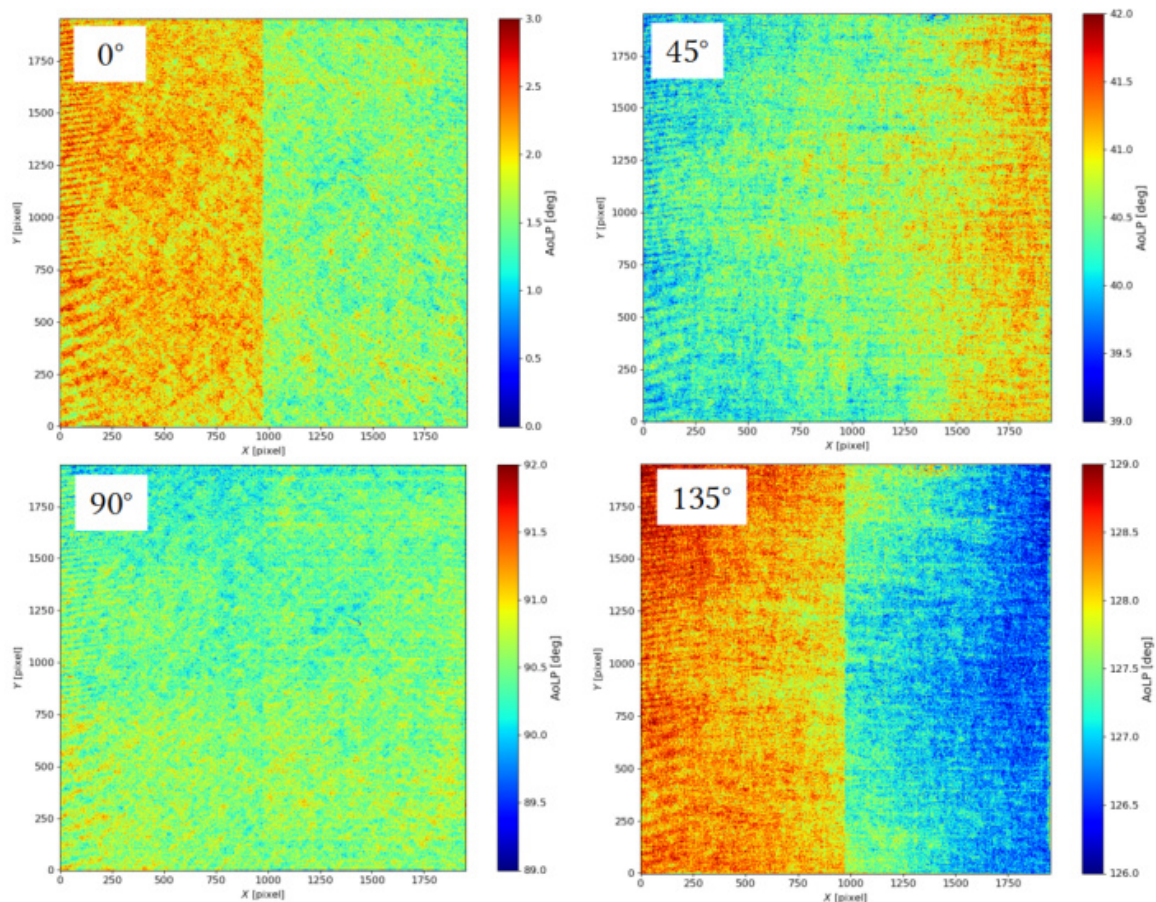

Figure 8. Measured Angle of Linear Polarisation (AoLP) for different polarised incoming light using the theoretical demodulation tensor. In particular, in this figure we show the 4 angles of $0^{\circ}, 45^{\circ}, 90^{\circ}, 135^{\circ}$.

\subsection{Micro-polarizers orientation and demodulation tensor}

As show in the previous Section, the degrees and angles of linear polarisation results to be not much consistent with the expected theoretical ones. To improve these results we need to consider a demodulation tensor different from the theoretical one. This new demodulation tensor must take into account different aspects not considered in the theoretical $\mathbf{X}^{\dagger}$, like, for example, the effective orientation of each micro-polarizer and other characteristics 

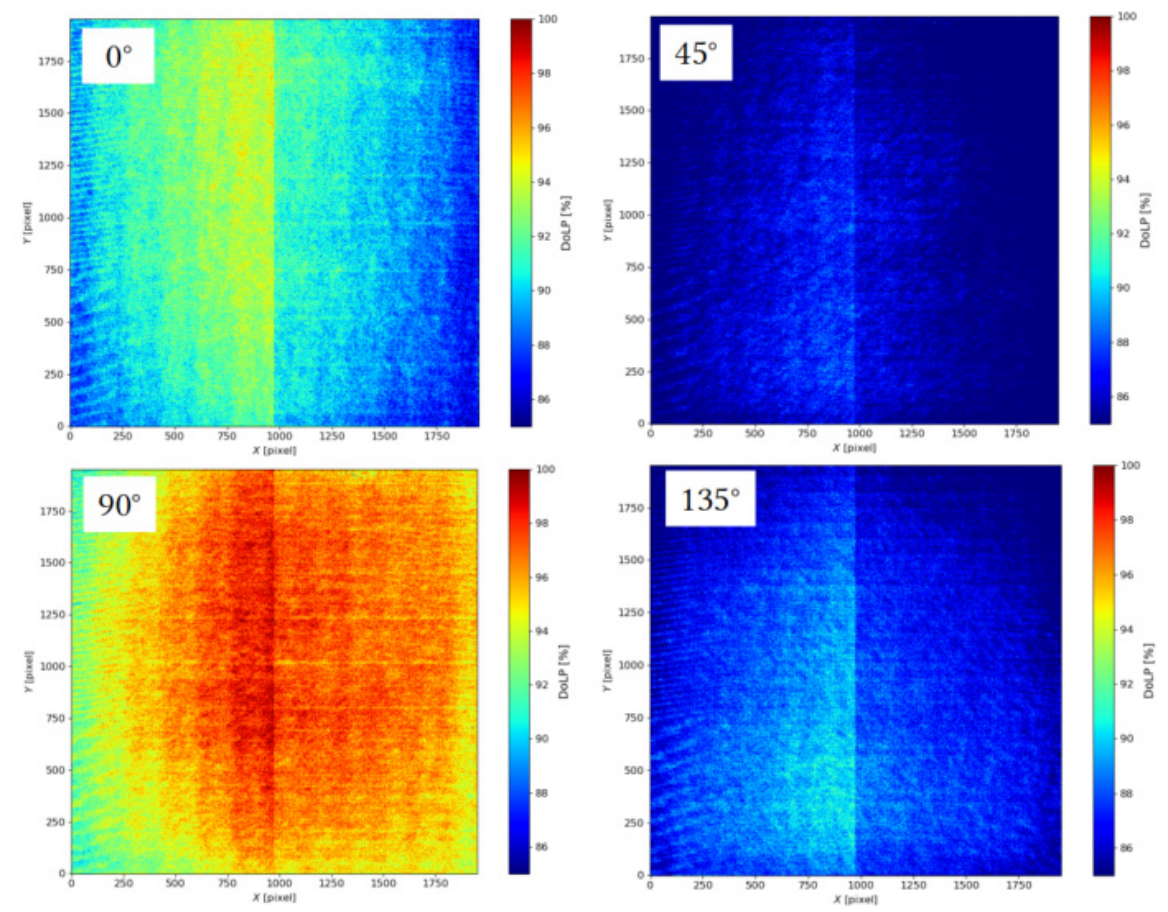

Figure 9. Measured Degree of Linear Polarisation (DoLP) for different polarised incoming light using the theoretical demodulation tensor.

of the polarising elements upon each pixel. As first step, is useful to point out that, exactly like for the study of polarised light (Subsec. 1.1), to characterise the behaviour of a linearly polarising element, it is necessary to have three quantities as well. These quantities are:

- efficiency (i.e. its ability to reject the unwanted polarisation states)

- throughput

- position angle

There are different conventions commonly used to present these three quantities. Considering I, Q, and U as the Stokes parameters polarimetrically describing the incident light beam, in this work we adopt the convention that the output beam intensity passing through a polarising element is given by: ${ }^{4,5}$

$$
S_{k}=A_{k} I+\epsilon_{k}\left(B_{k} Q+C_{k} U\right)
$$

where $S_{k}$ is the measured signal, $A_{k}, B_{k}, C_{k}$ are transmissivity terms and $\epsilon_{k}$ the polarising efficiency that describe each polarizer (i.e., each pixel of the PolarCam). The subscript $k$ denotes the polarizer orientation. Considering Eqs. 2 and 3, the above equation can be rewritten as:

$$
S_{k}=A_{k} I+\epsilon_{k}\left(B_{k} I p \cos 2 \psi+C_{k} I p \sin 2 \psi\right)
$$

where $\mathrm{I}$ is the total intensity, $p$ is the intrinsic fractional polarisation of the source and $\psi$ is the intrinsic polarisation angle. The transmissivity terms can now be written in terms of a generic throughput $t_{k}$ :

$$
S_{k}=\frac{1}{2} t_{k} I+\frac{1}{2} t_{k} \epsilon_{k} I p \cos 2 \phi_{k} \cos 2 \psi+\frac{1}{2} t_{k} \epsilon_{k} I p \sin 2 \phi_{k} \sin 2 \psi
$$


where $\phi_{k}$ are the position angles of the polarizers. It is possible to simplify the equation by set $p=1$. In this way, equation results to be:

$$
S_{k}=\frac{1}{2} t_{k} I\left[1+\epsilon_{k}\left(\cos 2 \phi_{k} \cos 2 \psi+\sin 2 \phi_{k} \sin 2 \psi\right)\right]
$$

By fitting to the response curves it is possible to determine the efficiency $\epsilon_{k}$ and orientation $\phi_{k}$ of every (micro)polarizer. This fit was performed for each PolarCam pixel, since each pixel correspond to a different micropolarizer. The transmissivity $t_{k}$ was evaluated before the data fitting. These values have been obtained searching the pixel with the highest transmissivity with the same input, considered as $1 / 2\left(S_{k}^{\psi_{0}}+S_{k}^{\psi_{45}}+S_{k}^{\psi_{90}}+S_{k}^{\psi_{135}}\right)$, and normalising the others $t_{k}$ in function of it. An example of what we obtain from a fit is shown in Fig. 10. This study was performed setting the camera analog gain $\mathrm{AG}=(44,44)$, the digital gain $\mathrm{DG}=1$ and and exposure time of $t_{e x p}=71.36 \mathrm{~ms}$. In particular, instead of $S_{k}$, we considered a normalised irradiance $\mathrm{S}$ defined as:

$$
S=\frac{S_{k}}{\frac{1}{2}\left(S_{k}^{\psi_{0}}+S_{k}^{\psi_{45}}+S_{k}^{\psi_{90}}+S_{k}^{\psi_{135}}\right)}
$$

where $k=\left[0^{\circ}, 45^{\circ}, 90^{\circ}, 135^{\circ}\right]$ are the micropolarizers orientations.

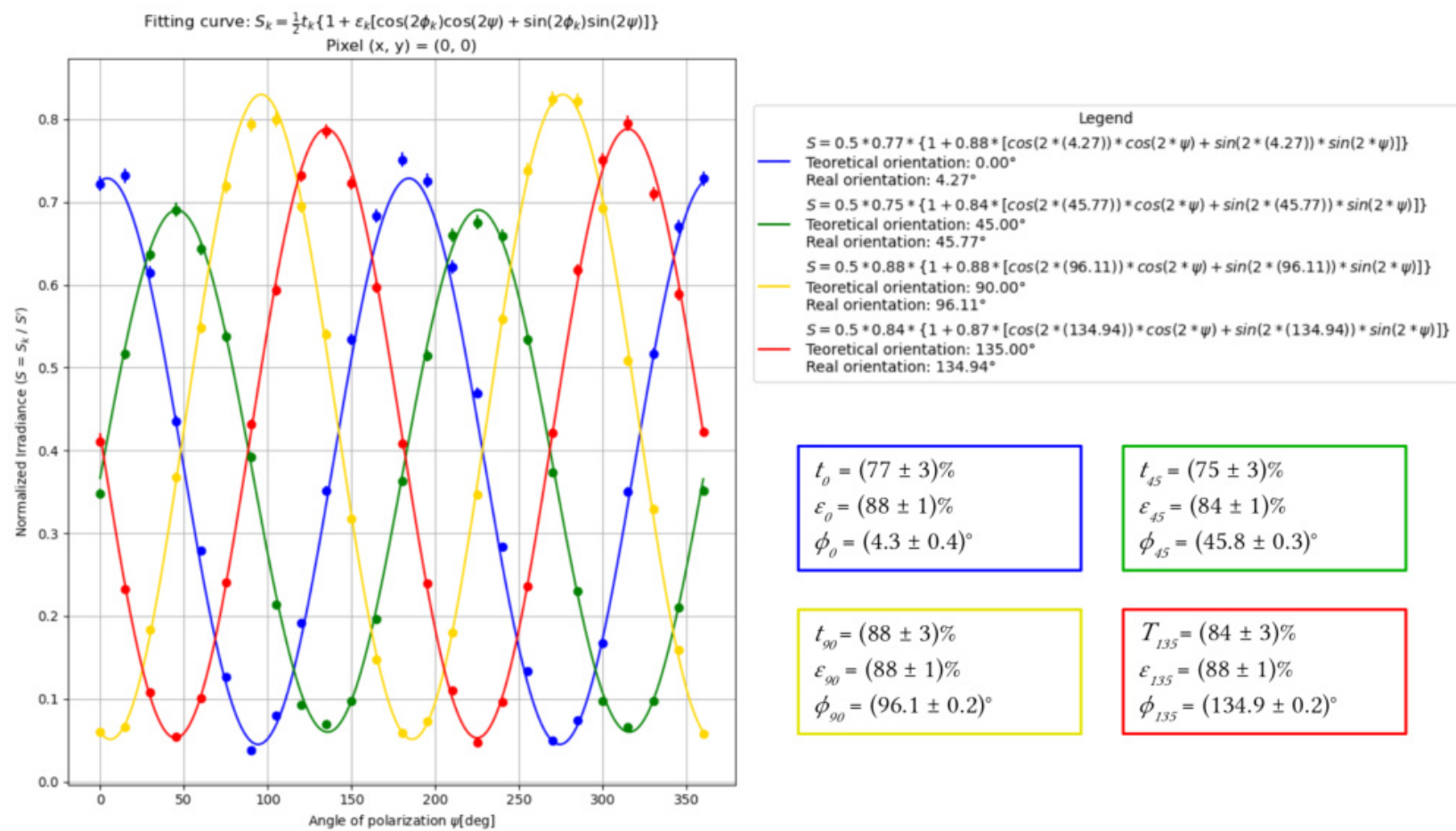

Figure 10. Example of curve fit for a particular super-pixel [in this case, the super-pixel associated to the pixel $(0,0)$ ]. It is possible to see that the obtained values for the $\phi_{k}$ parameters are pretty consistent with the expected ones.

At that point is possible to estimate the Stokes parameters of the incident light. To do that, as illustrated in Subsec. 1.1, we have to solve the following equation:

$$
\mathbf{m}=\mathbf{X} \mathbf{S}_{\text {input }}
$$

where $\mathbf{m}$ is the vector of the images with the four different linear polarisation, $\mathbf{X}$ is the modulation matrix and $\mathbf{S}_{\text {input }}$ is the Stokes vector associated to the incoming light. In particular, considering the $\epsilon_{i}$ and $\phi_{i}$ obtained from the data fit, we get the matrix in Eq. 17. 


$$
\mathbf{X}=\frac{1}{2} t_{k}\left(\begin{array}{ccc}
1 & \epsilon_{1} \cos 2 \phi_{1} & \epsilon_{1} \sin 2 \phi_{1} \\
1 & \epsilon_{2} \cos 2 \phi_{2} & \epsilon_{2} \sin 2 \phi_{2} \\
1 & \epsilon_{3} \cos 2 \phi_{3} & \epsilon_{3} \sin 2 \phi_{3} \\
1 & \epsilon_{4} \cos 2 \phi_{4} & \epsilon_{4} \sin 2 \phi_{4}
\end{array}\right)=\frac{1}{2} t_{k}\left(\begin{array}{ccc}
1 & 0.898 & 0.148 \\
1 & -0.032 & 0.870 \\
1 & -0.890 & -0.188 \\
1 & 0.006 & -0.910
\end{array}\right)
$$

By (pseudo)inverting the $\mathbf{X}$ matrix it is possible to obtain the demodulation matrix $\mathbf{X}^{\dagger}$ :

$$
\mathbf{X}^{\dagger}=\left(\mathbf{X}^{T} \mathbf{X}\right)^{-1} \mathbf{X}^{T}=\left(\begin{array}{cccc}
0.619 & 0.572 & 0.542 & 0.485 \\
1.189 & -0.185 & -1.172 & 0.301 \\
0.070 & 1.301 & -0.017 & -1.303
\end{array}\right)
$$

Then, from Eq. 16 and Eq. 18 we can obtain $\mathbf{S}_{\text {input }}$ as:

$$
\mathbf{X}^{\dagger} \mathbf{m}=\mathbf{S}_{\text {input }} \rightarrow\left(\begin{array}{cccc}
0.619 & 0.572 & 0.542 & 0.485 \\
1.189 & -0.185 & -1.172 & 0.301 \\
0.070 & 1.301 & -0.017 & -1.303
\end{array}\right)\left(\begin{array}{c}
I M G_{0} \\
I M G_{45} \\
I M G_{90} \\
I M G_{135}
\end{array}\right)=\left(\begin{array}{c}
I_{\text {input }} \\
Q_{\text {input }} \\
U_{\text {input }}
\end{array}\right)
$$

Performing this process for the entire frame (pixel by pixel) we obtain a demodulation tensor $\mathbf{X}^{\dagger}$ where each of the 12 elements is a matrix (Fig. 11). A summary of this process is shown in Fig. 12.
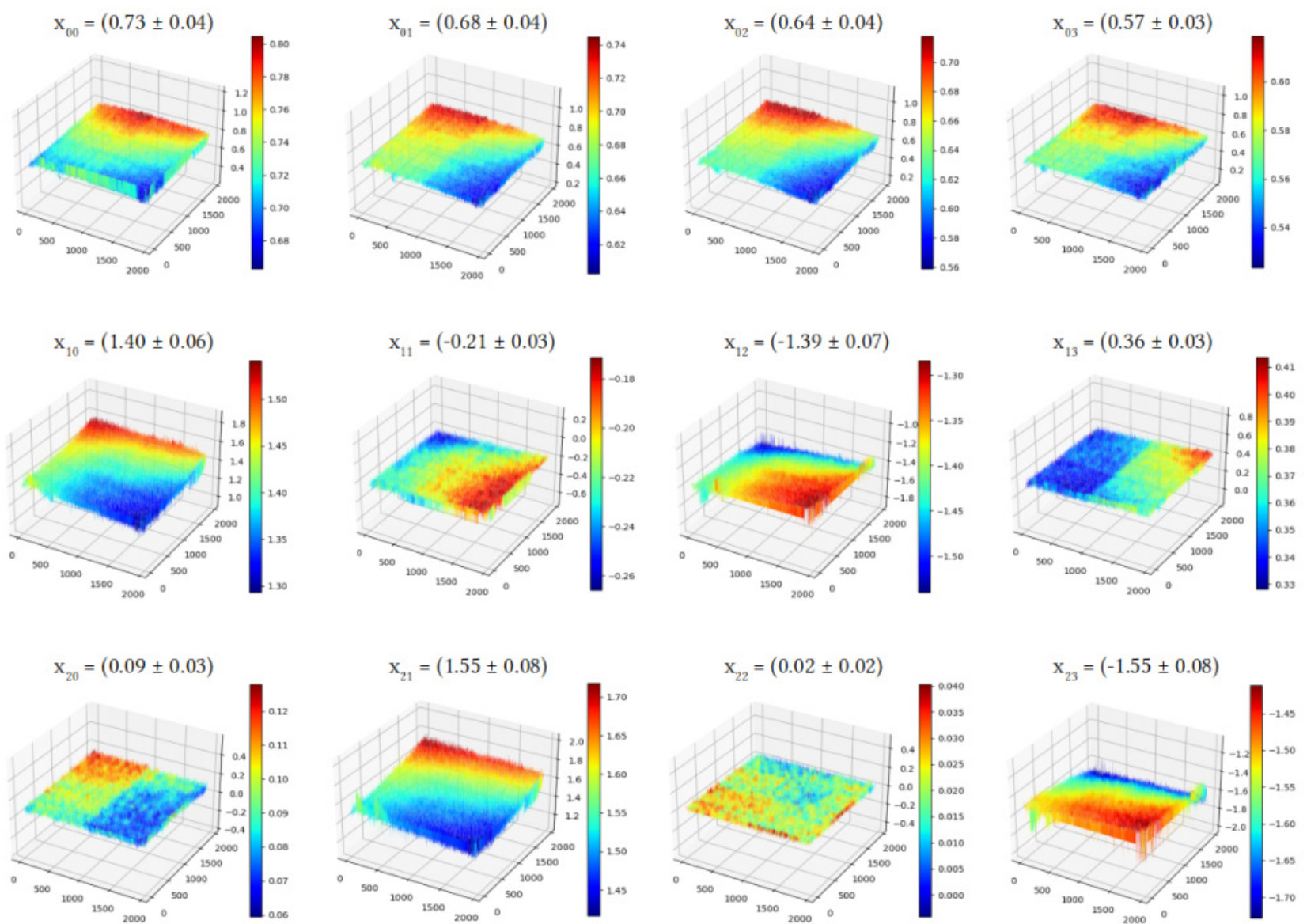

Figure 11. Demodulation tensor element $x_{i j}$ obtained during PolarCam calibration. 

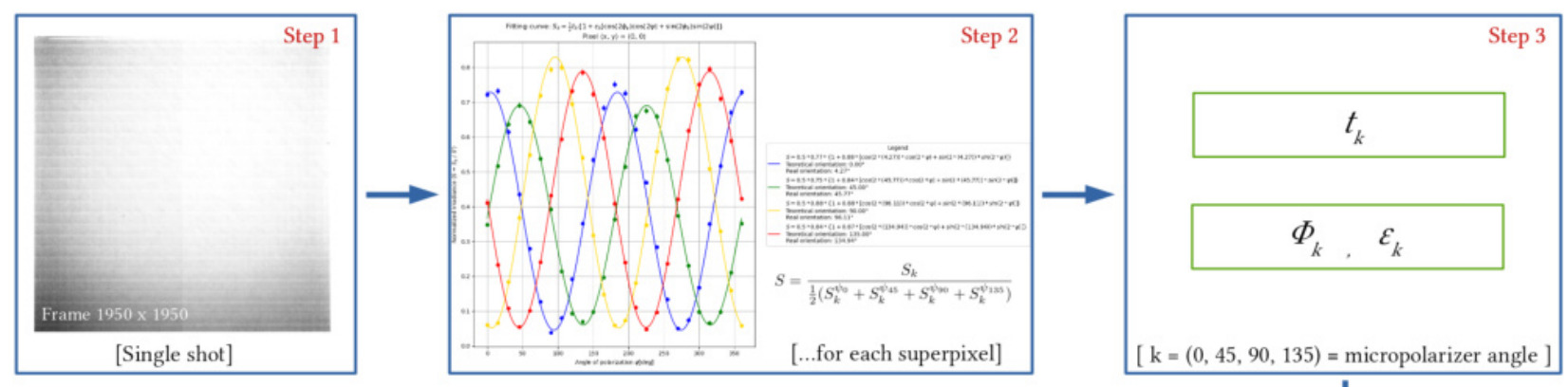

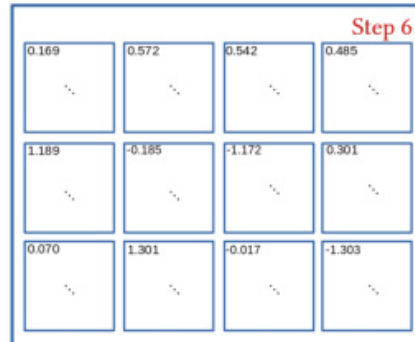

[ Demodulation tensor ]

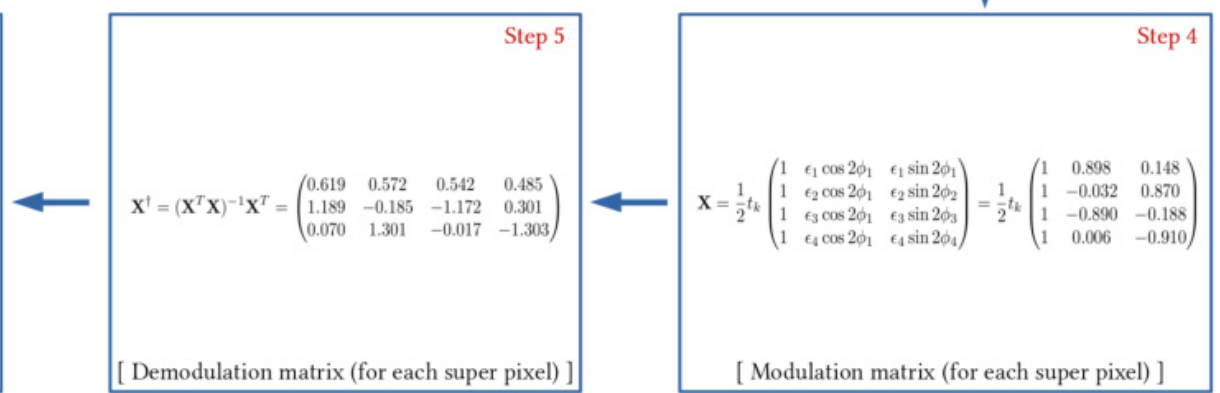

Figure 12. Schematic step-by-step procedure for the experimental evaluation of the demodulation tensor $\mathbf{X}^{\dagger}$.

Using this specific demodulation tensor (specific for our camera), we obtain, using the same procedure already applied for the theoretical demodulation tensor (Subsec. 3.3), the Stokes parameters, the DoLP and the AoLP. The results for the unpolarized light case (flat-field source) are shown in Fig. 13. In Fig 14 and Fig 16 are depicted the results for the polarised light. As expected, the use of a calibrated demodulation tensor improves significantly the accuracy in the measurements of the polarisation state of the light detected by the PolarCam - In particular, it is possible to see how the new demodulation tensor remove (almost totally) the residual instrumental polarisation. Moreover, the frames acquired with the polarimetric flat-field, show an almost perfect agreement between the flat-field polarizer orientation and the measured one (the differences between the expected angles of linear polarisation and the obtained ones are shown in Fig. 15).

A summary and comparison of these results is reported in Tab. 4. Finally, in Fig. 17, we show the maps for the different micropolarizers throughput $t_{k}$ for the 4 orientations (the same study was performed for the efficiency $\epsilon_{k}$ as well). Moreover, looking at Fig. 18, it is possible to see that the throughput seems to be systematically different for pixels with different orientations. In Fig. 19, a map of the effective micro-polarizer orientation is shown.

Table 4. Unpolarised flat-field (UFF) and polarimetric flat-field (PFF) DoLP and AoLP retrieved by applying the calibrated demodulation tensor.

\begin{tabular}{|c|c|c|c|c|}
\hline Set up & Expected DoLP & Obtained DoLP & Expected AoLP & Obtained AoLP \\
\hline $\mathrm{UFF}$ & $0 \%$ & $(2 \pm 2) \%$ & $/$ & $/$ \\
$\mathrm{PFF}\left(0^{\circ}\right)$ & $100 \%$ & $(100 \pm 2) \%$ & $0^{\circ}$ & $\left(0.0^{\circ} \pm 0.5^{\circ}\right)$ \\
$\mathrm{PFF}\left(45^{\circ}\right)$ & $100 \%$ & $(100 \pm 2) \%$ & $45^{\circ}$ & $\left(44.6^{\circ} \pm 0.5^{\circ}\right)$ \\
$\mathrm{PFF}\left(90^{\circ}\right)$ & $100 \%$ & $(99 \pm 2) \%$ & $90^{\circ}$ & $\left(90.0^{\circ} \pm 0.5^{\circ}\right)$ \\
$\mathrm{PFF}\left(135^{\circ}\right)$ & $100 \%$ & $(100 \pm 2) \%$ & $135^{\circ}$ & $\left(135.2^{\circ} \pm 0.5^{\circ}\right)$ \\
\hline
\end{tabular}



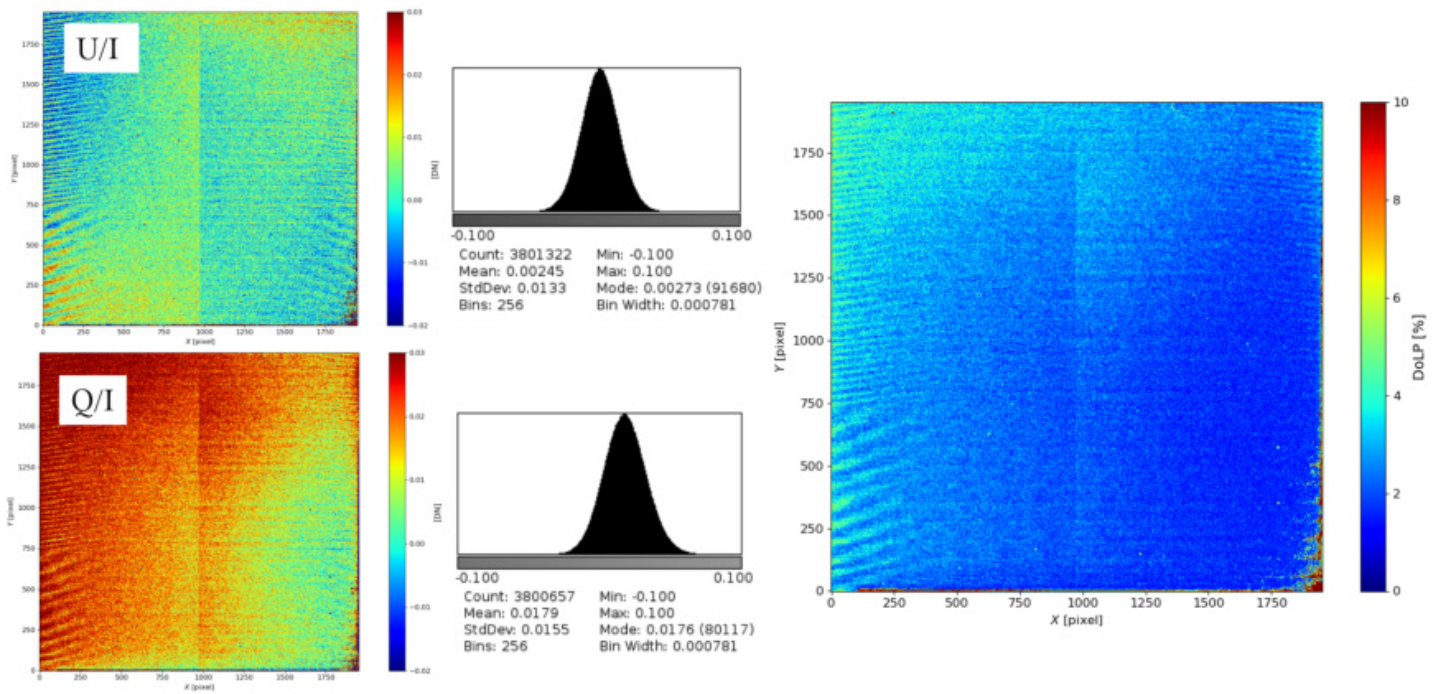

Figure 13. Test with flat-field (unpolarised light). Left side: Stokes parameters obtained with the calibrated demodulation tensor (Stokes vector $=[1, \mathrm{Q} / \mathrm{I}, \mathrm{U} / \mathrm{I}]$ ). Right side: Degree of Linear Polarization obtained with the calibrated demodulation tensor.
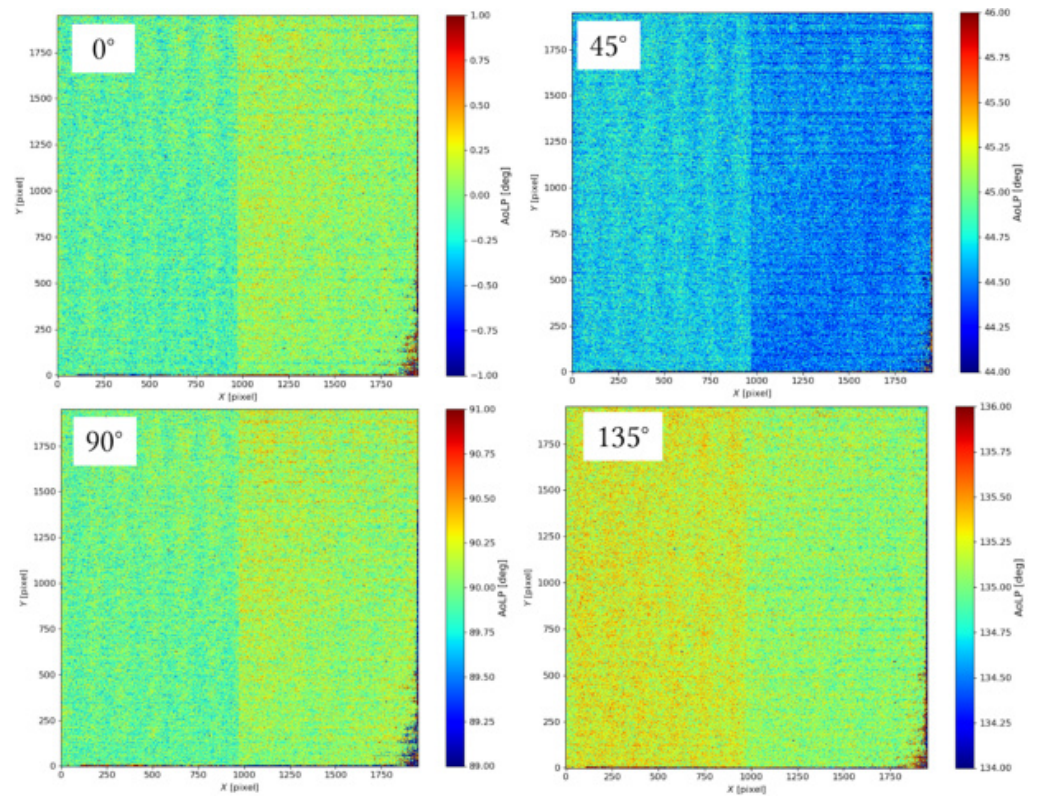

Figure 14. Measured Angle of Linear Polarization (AoLP) for different polarized incoming light using the calibrated demodulation tensor. In particular, in this picture, the results for the angles of $0^{\circ}, 45^{\circ}, 90^{\circ}, 135^{\circ}$ are shown.

\section{APPLICATION OF THE POLARCAM IN A SOLAR CORONAGRAPH}

The Sun has a million-degree atmosphere that extends across the solar system. The outermost layer of the solar atmosphere is the solar corona. The solar corona is mainly photospheric light scattered by the electrons (and other particles/dust). The electrons scattering, known as "Thomson scattering", is linearly polarised and it produces what is called K-corona. Instead, the photospheric radiation scattered everything else is unpolarized and is called F-corona. In particular, we are interested in the K-corona (as several other relevant physical parameters can be extrapolated from it). It is therefore necessary to carry out polarimetric studies and, to do that, we used a PolarCam. 


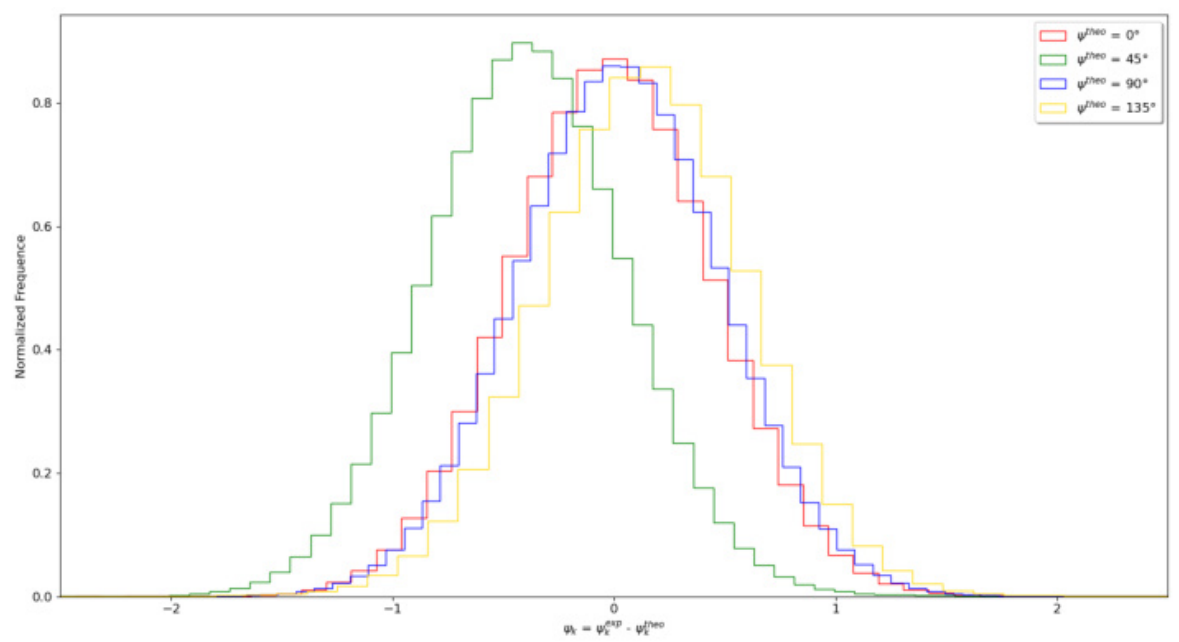

Figure 15. Histograms of the differences between the measured Angle of Linear Polarization $\left(\psi^{\exp }\right)$ and the orientation of the acceptance axis of the pre-polarizer $\left(\psi^{\text {theo }}\right)$.
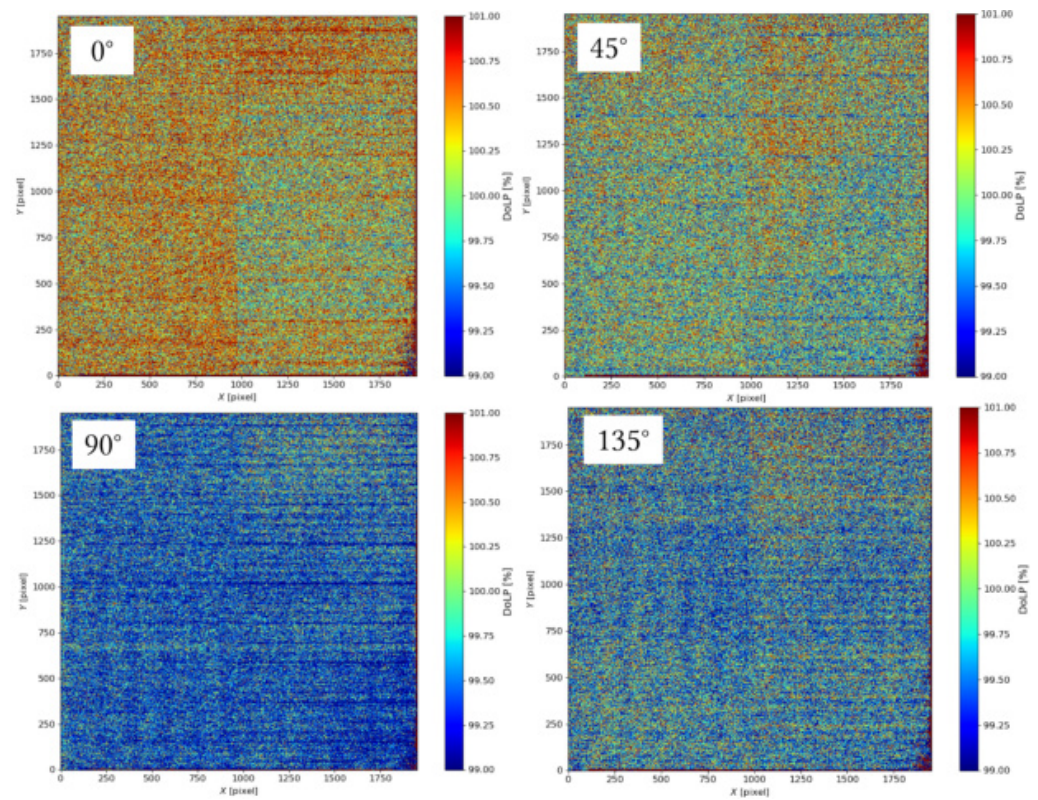

Figure 16. Measured Degree of Linear Polarization (DoLP) for different polarised light using the calibrated demodulation tensor.

However, a particular telescope is required. Indeed, the brightness of solar corona decrease exponentially moving away from the solar photosphere. In particular, even at a few solar radii, its brightness is about $10^{6}$ times lower. This means that, in order to observe the corona, it is necessary to hide the solar disk to avoid to be "blinded" by it (for this reason it is possible to observe the solar corona with the naked eye only during a total solar eclipse). Special telescopes called coronographs are used for the purpose. The coronographs has an occulter that has the same function of the Moon during a total solar eclipse. Moreover, the ground-based observations must consider that, since the sky scatter the solar light, it is also necessary to have a particularly clear sky. In particular, it is necessary a so called "coronagraphic sky"; a sky with a brightness at the order of $\sim 10^{-6}\left[\mathrm{~B}_{\odot}\right]$ or less. To date, the only place from the Earth that has these characteristics and where coronagraphic studies are performed continuously, is in Haleakala, in Maui, Hawai'i. 

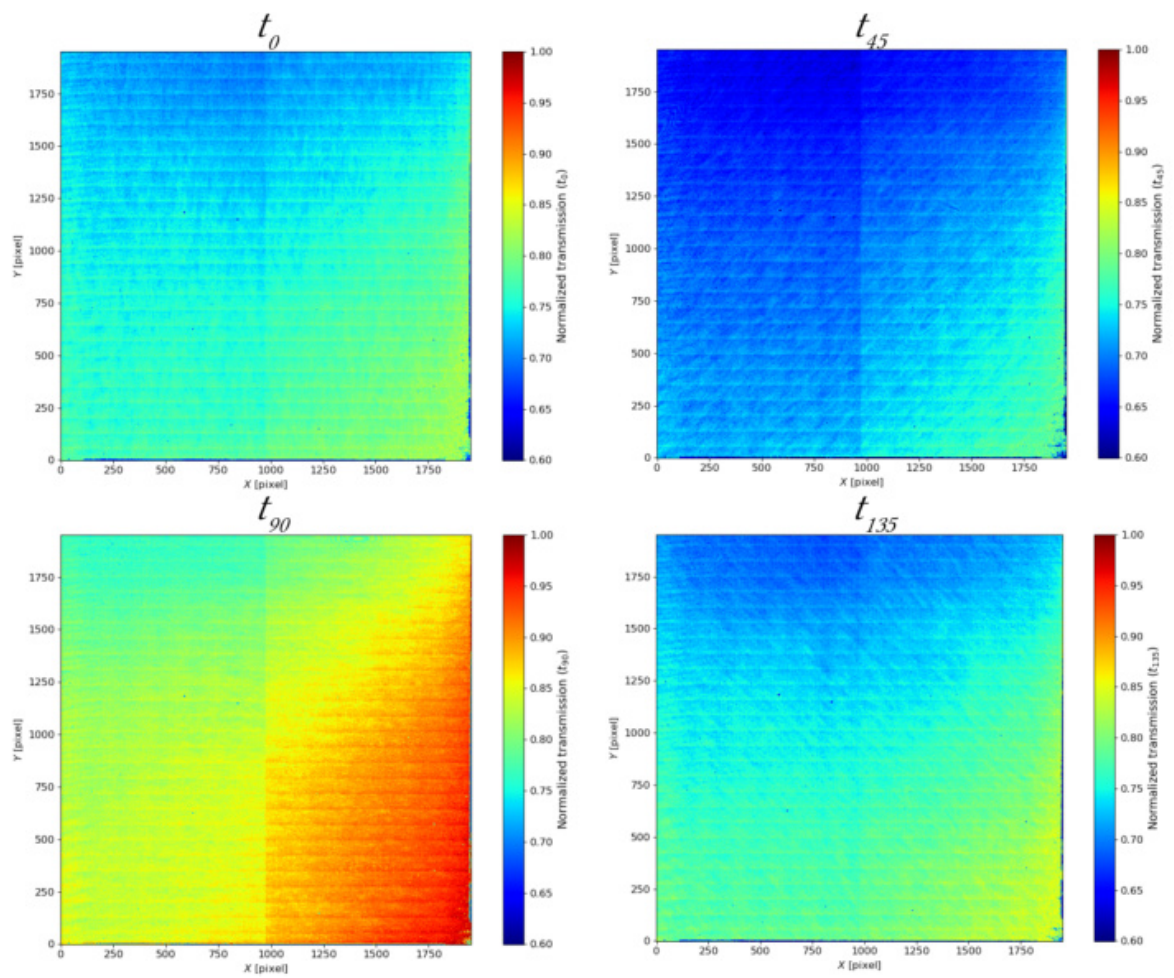

Figure 17. Throughput $t_{k}$ (for each pixel orientation) normalised to the maximum throughput of all pixels.

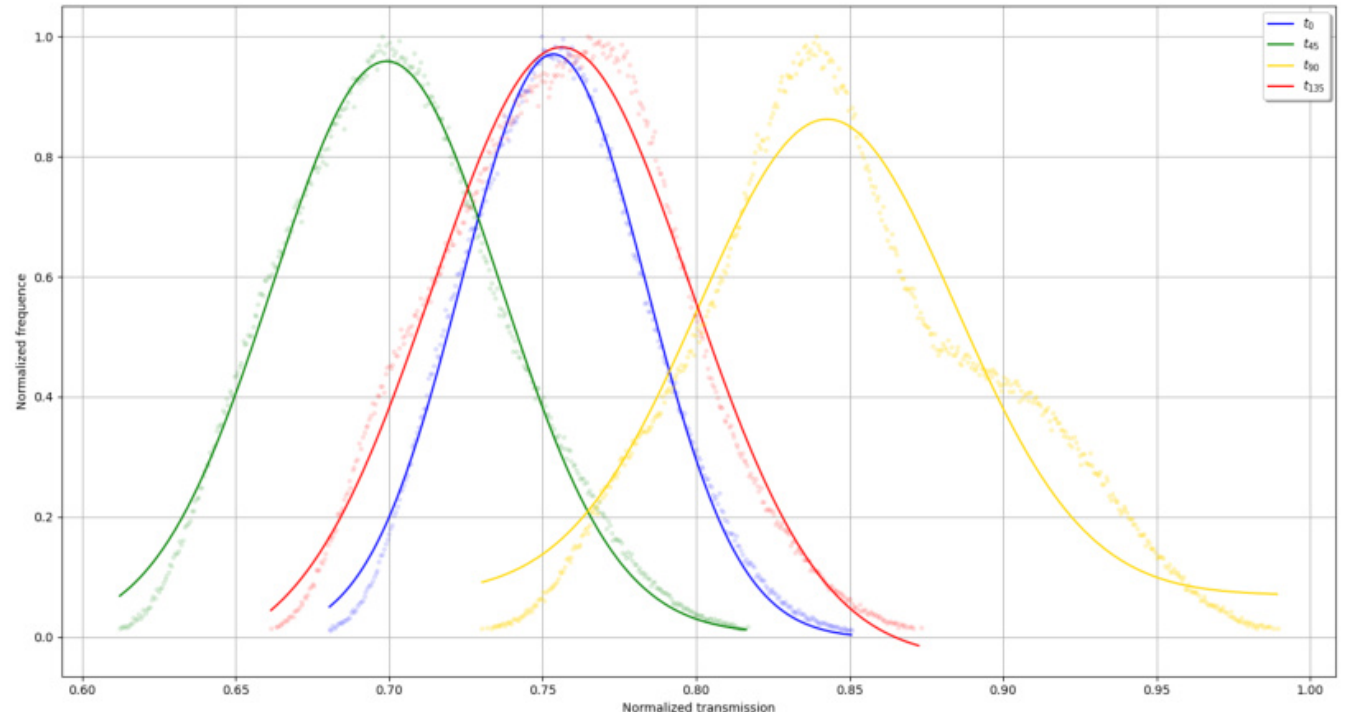

Figure 18. Histograms of throughput $t_{k}$ (for each pixel orientation) normalised to the maximum throughput of all pixels.

\subsection{ESCAPE Project}

The Antarctica plateau of Dome C (coord: $75^{\circ} 06$ 'S; $123^{\circ} 20^{\prime} \mathrm{E}$ ) offers the unique opportunity for ground-based observations of the solar corona thanks to the high altitude of the site $(3,233 \mathrm{~m}$ a.s.l.), the almost total absence of human pollution and the uninterrupted daily hours for observations during the antarctic summer. ${ }^{6}$ In order to take advantage of this opportunity, the Italian Piano Nazionale Ricerche Antartico (PNRA) ${ }^{7}$ has selected our 

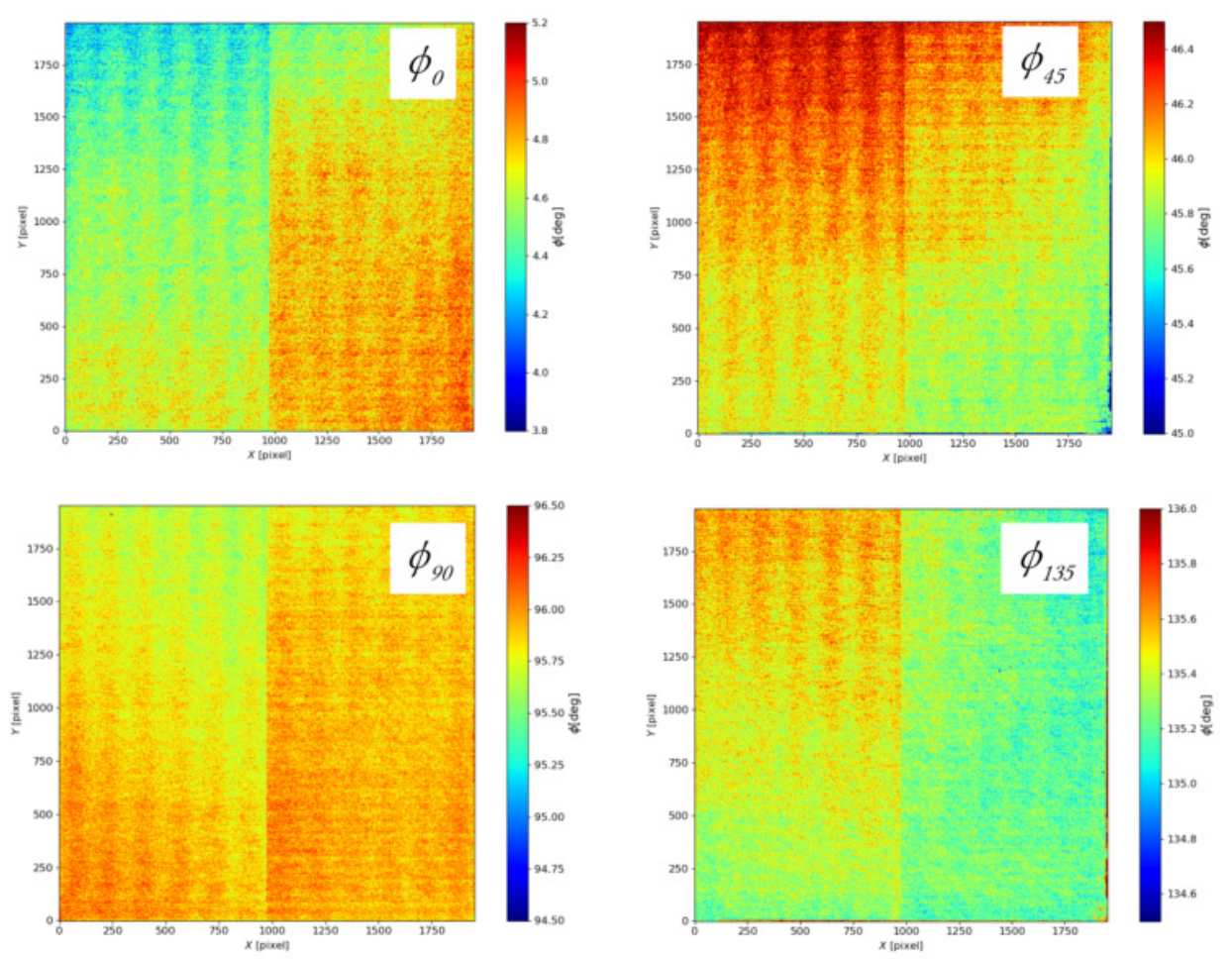

Figure 19. Maps of the micro-polarizer orientations, $\phi_{k}$.

proposal for the "Extreme Solar CoronagraphyAntarctic Program Experiment" (ESCAPE) for the installation of an Antarctic coronagraph (AntarctiCor) at the Italian-French Concordia base at Dome C. More information about ESCAPE Project and its scientific objectives can be found in Ref 8 .

\subsection{Antarctic Coronagraph}

The Antarctica solar coronagraph (AntarctiCor) for the ESCAPE program is a classical internally-occulted Lyot coronagraph (Fig. 20) based on the optical design of the ASPIICS coronagraph of the PROBA-3 ESA mission. ${ }^{9}$ The chosen detector for this telescope is the PolarCam. The main features of the instrument are summarized in Tab. 5 .

Before the Antarctic campaigns, instrument calibrations were carried out. However, being this paper not specifically dedicated to the AntarctiCor, only the aspects of the calibration concerning the camera will be treated here.

\subsubsection{Point Spread Function}

To evaluate the Point Spread Function (PSF) we used a pinhole $(50 \mu \mathrm{m})$ on the Illumination System Visible Light (ISVL) ${ }^{11}$ in the Optical Payload System - OPSys - Facility ${ }^{12}$ in ALTEC, Turin, Italy. In particular, 10 images are summed to increase the SNR. The image region with the pinhole (Fig. 21) is selected as Region Of Interest (ROI) to evaluate the PSF. For these measurements, the following settings of the PolarCam were used: Exposure time equal to $71.82 \mathrm{~ms}$ and digital gain equal to 16 . Then, for the two dimensions, rows (red) and columns (blue), the, pixel values are plotted and a best-fit ("horizontal" and "vertical" fit respectively) has been performed with a Gaussian function. The results are shown in Fig. 22.

From the variance is possible to obtain the Half Width at Half Maximum (HWHM) for both the fit, obtaining: $H W H M_{h}=\sqrt{2 \cdot \log 2} \cdot \sigma_{h}=1.38 \pm 0.05$ and $H W H M_{v}=\sqrt{2 \cdot \log 2} \cdot \sigma_{v}=1.36 \pm 0.06$ respectively. Averaging between them, we obtain: $\overline{H W H M}=1.37 \pm 0.04$ pixels. Then, considering the Full Width at Half Maximum (FWHM) we can considered that a "point-like signal" is spread over $\overline{F W H M}=2.74 \pm 0.08$ pixels $(\equiv 20.28 \mu \mathrm{m})$. 
Table 5. Main AntarctiCor features. ${ }^{8}$

\begin{tabular}{|l|l|}
\hline Telescope design & Classic internally-occulted Lyot coronagraph ${ }^{10}$ \\
Aperture & $50 \mathrm{~mm}$ \\
Eff. Focal Length & $700 \mathrm{~mm}$ \\
f/ratio & 14 \\
Spectral Ranges & $(591 \pm 5) \mathrm{nm}$ \\
Camera type & Interline transfer CCD PolarCam (model: U4) $)^{1}$ \\
Camera format & $1950 \times 1950$ pixels \\
Pixel size & $7.4 \mu \mathrm{m} \times 7.4 \mu \mathrm{m}$ \\
Plate scale & 4.3 arcsec/pixel \\
Field of View & $\pm 0.6^{\circ} \equiv \pm 2.24 \mathrm{R}_{\odot}$ \\
Polarization analysis & spatial modulation by linear micropolarizer array on CCD sensor \\
\hline
\end{tabular}
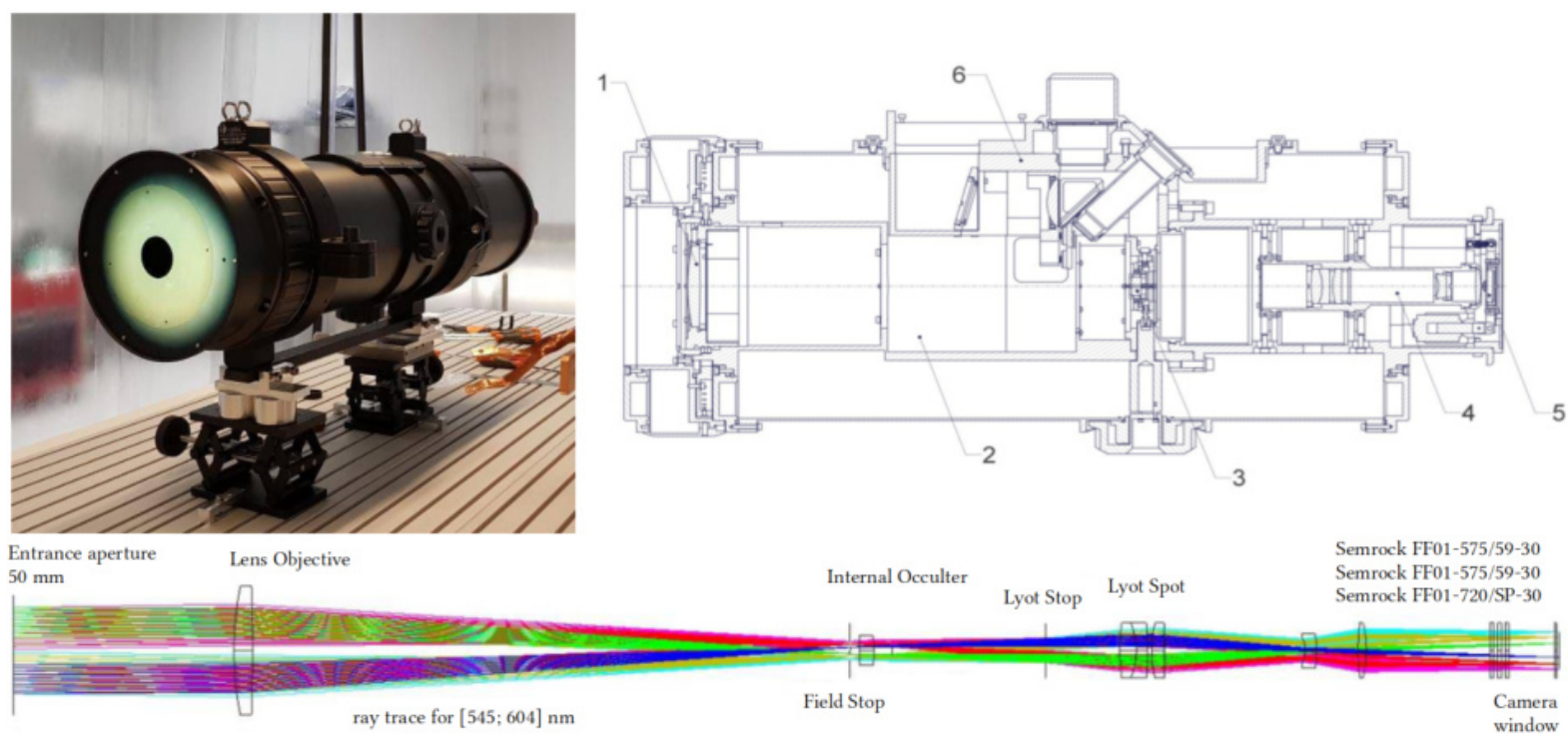

Figure 20. Up: AntarctiCor in the INAF Optical Payload Systems facility (OPSys), Turin-Italy, for tests and calibrations. In the right panel, the main sub-assembly is shown. In particular it contains the following items: 1. Objective lens assembly; 2. Inner main barrel assembly; 3. Internal Occulter assembly; 4. Lenses assembly; 5. Filter assembly; 6 . Light trap assembly; 7. Microscope assembly. Down: AntarctiCor optical ray trace.

Looking at Tab. 5 we can see that the wavelength $\lambda=591 \mathrm{~nm}$ and the f-ratio $\mathrm{F} / \#=14$. Then we can evaluate the telescope diffraction limit:

$$
\text { Spot size }[\mu \mathrm{m}]=1.22 * \lambda[\mu \mathrm{m}] * F / \# \approx 10.1 \mu \mathrm{m}
$$

Then, we can conclude that the telescope is diffraction limited. Anyhow, it is good to remember that, being the super pixel composed by 4 different orientation (whereas each pixel has dimension $7.4 \times 7.4 \mu \mathrm{m}$ ), the pixel dimension for a frame post-demosaicing (i.e. with a single polarizer orientation; see Fig. 2) results to be twice the original frame: $14.8 \times 14.8 \mu \mathrm{m}$.

\subsection{Antarctic campaign}

During the Antarctic campaign, in addition to coronal images (still under analysis), systematic images of the sky were acquired. The goal is to study it from a polarimetric point of view and try to evaluate its brightness. 


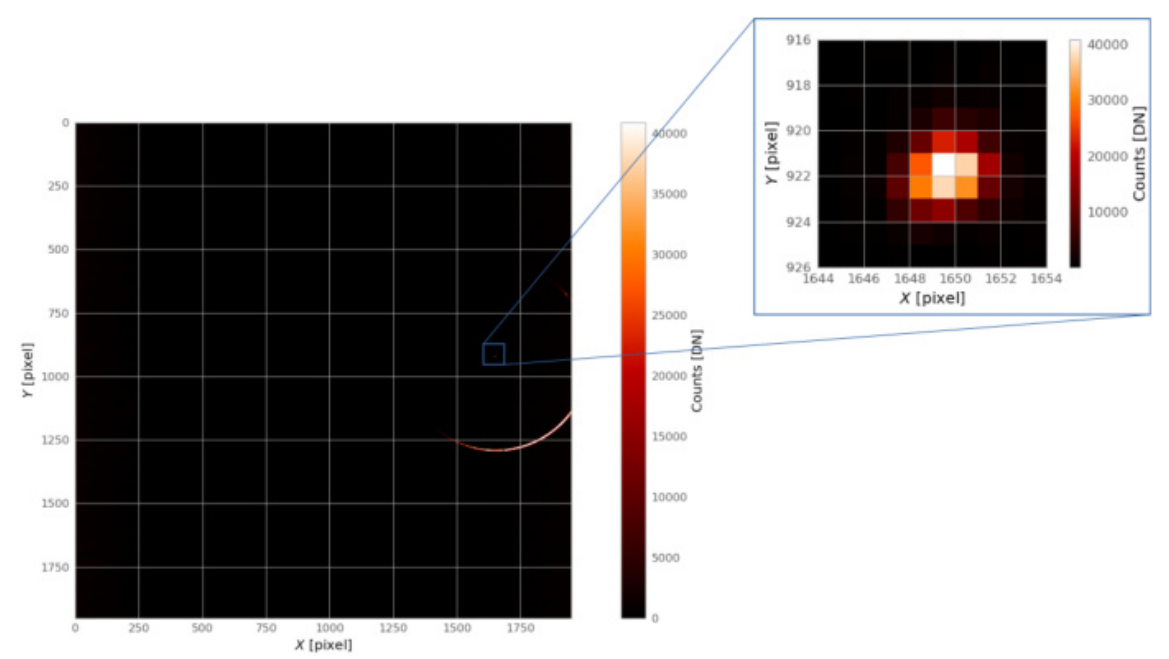

Figure 21. Sum of 10 pinhole images with region of interest (ROI). A light leak from the edge of the pinhole mask is also visible around the region $[1750,1250]$ as a thin line.
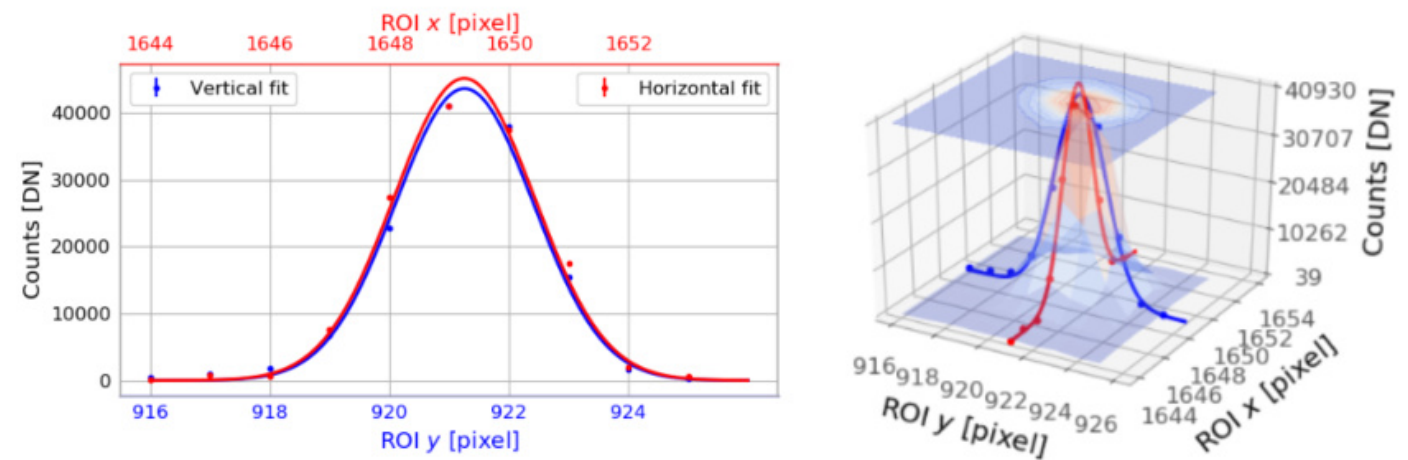

Figure 22. Gaussian best-fit of the ROI for rows and columns. On the right side, a 3D representation of the considered fit-geometry. The error bars are negligible and not visible in the plots.

In Fig. 23 an example of the Intensity (Stokes I parameter) of the data acquired from the sky and from the Sun with an opal (i.e., diffuser) in front of the telescope are shown.

The sky brightness $\left(\mathrm{B}\left[\mathrm{B}_{\odot}\right]\right)$ was measured for the full data acquisition campaign at almost regular intervals during the day. To obtain the sky brightness in unit of solar disk brightness $\left(B_{s k y}\left[\mathrm{~B}_{\text {odot }}\right]\right)$ a "sun-centered + diffuser" $\left(I_{\text {opal }}\right)$ and a sky $\left(I_{s k y}\right)$ image are needed. Indeed, from the ratio of these quantities we can obtain:

$$
\frac{\bar{I}_{s k y}}{\bar{I}_{d i f f}}=\frac{I_{s k y} / t_{e x p}^{s k y}}{I_{d i f f} / t_{\text {exp }}^{\text {diff }}}=\frac{B_{\text {sky }} / t_{\text {exp }}^{\text {sky }}}{\left(B_{\odot} \cdot T_{\text {diff }}\right) / t_{\text {exp }}^{\text {diff }}}
$$

whereas $t_{\text {exp }}^{i}$ are the exposure times. Then, multiplying by the diffuser transmissivity $T_{\text {diff }}(\sim 28 \%):^{13}$

$$
\frac{\bar{I}_{s k y}}{\bar{I}_{d i f f}} \cdot T_{d i f f}=\frac{B_{s k y} / t_{e x p}^{s k y}}{\left(B_{\odot} \cdot T_{d i f f}\right) / t_{e x p}^{d i f f}} \cdot T_{d i f f}=\frac{B_{s k y} / t_{e x p}^{s k y}}{B_{\odot} / t_{e x p}^{d i f f}}
$$

Then, considering geometric factor $K=1.82 \times 10^{-5}$ (light scattered over the solid angle by the diffuser) it is 

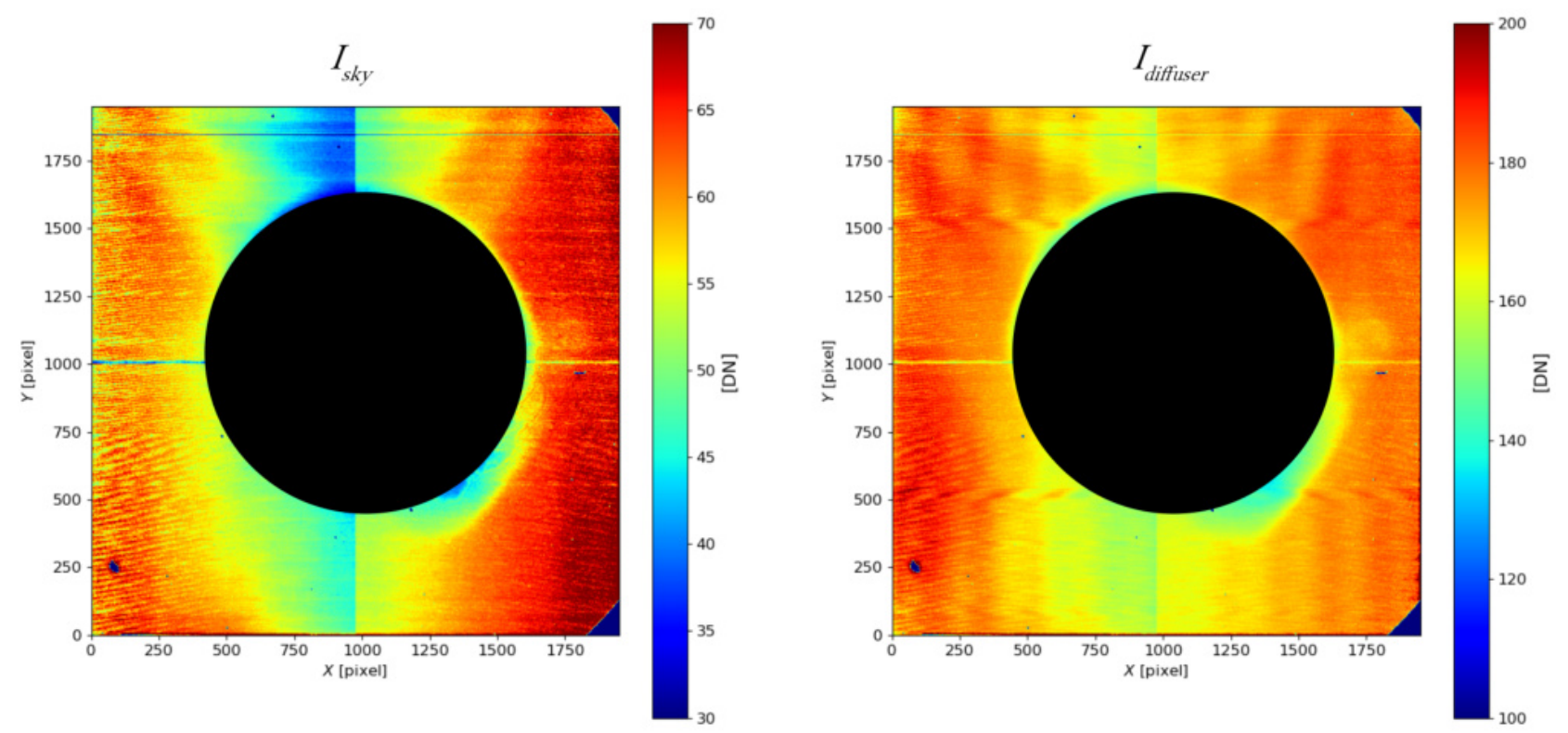

Figure 23. Left: Example of first Stokes parameter (i.e., I) obtained from an image acquired pointing to the sky. Right: Example of first Stokes parameter (i.e., I) obtained from an image acquired pointing to the Sun with a diffuser in front of the coronagraph.

possible to evaluate the $B_{s} k y\left[\mathrm{~B}_{\odot}\right]$ as:

$$
B_{s k y}\left[B_{\odot}\right]=\frac{\left(I_{s k y} / t_{e x p}^{s k y}\right)}{\left(I_{d i f f} / t_{e x p}^{d i f f}\right)} K T_{d i f f}
$$

Moreover, the obtained $B_{s k y}\left[B_{\odot}\right]$ frame was divided in four different pad and the final B was obtained by averaging them (Fig. 24).

A summary of the obtained sky brightness for different days and different hours is shown in Fig. 25. In particular, averaging over the different values and considering also that the $I_{s k y}$ results to be at the limit of the camera sensitivity $(\sim 10 \mathrm{DN})$, we get that: $\bar{B}_{s k y} \leq(1.17 \pm 0.03) \times 10^{-6} \mathrm{~B}_{\odot}$. This means that Dome $\mathrm{C}$ sky can be considered as "coronagraphic" sky.

\section{CONCLUSION}

This paper gives a description and shows the main features of a microarray polarizer camera with a particular focus on the PolarCam (mod. U4). In particular, the evaluation of the degree of linear polarisation seems to show an intrinsic polarisation using a theoretical demodulation tensor. However, both the degree and the angle of linear polarisation behaves as expected if a calibrated demodulation tensor is considered. A methodology for the polarimetric characterisation of these cameras is provided in the paper. A check on the correct micropolarizer orientation was also performed showing that the actual orientation is close to the nominal one. Maps of the micro-polarizers main characteristics are shown as well. Finally, an example of the application of these devices in the filed of solar coronagraphy is shown. As example, the brightness of the sky at Concordia Base, Dome $\mathrm{C}$ - Antarctica is evaluated from the ESCAPE project. A $\bar{B}_{s k y}=(1.17 \pm 0.03) \times 10^{-6} \mathrm{~B}_{\odot}$ has been measured during the XXXV italian expedition to Antarctica. This result demonstrate the quality of the DOME C site for coronagraphic measurements. 


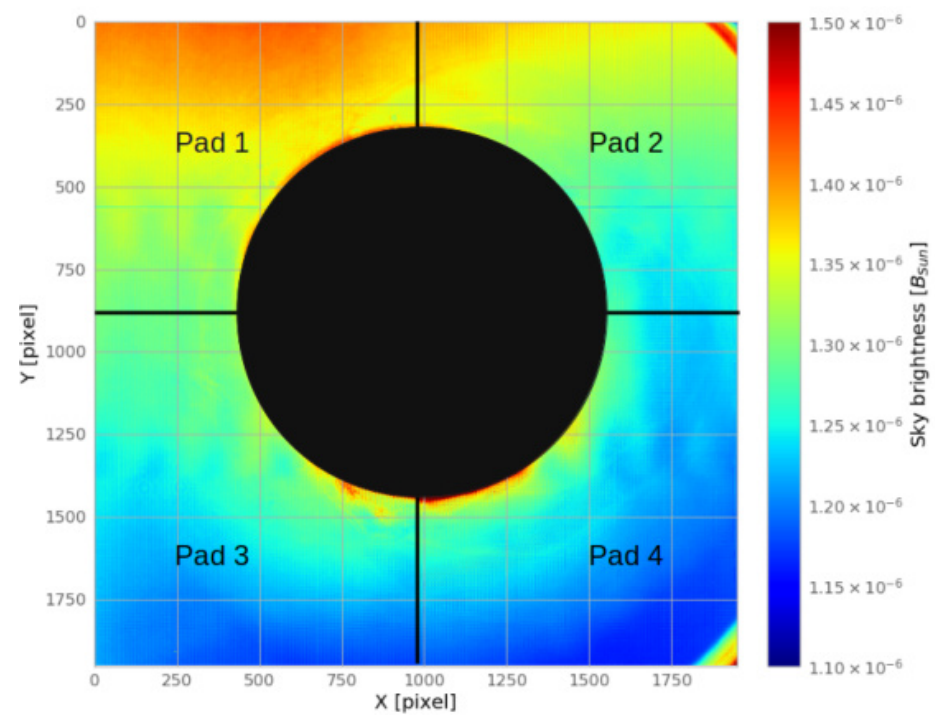

Figure 24. Example of the measured sky brightness (Dome C, Concordia Base - Antarctica). As shown, the entire frame is divided into 4 different pads. The final sky brightness is obtained as the average of the 4 pads.

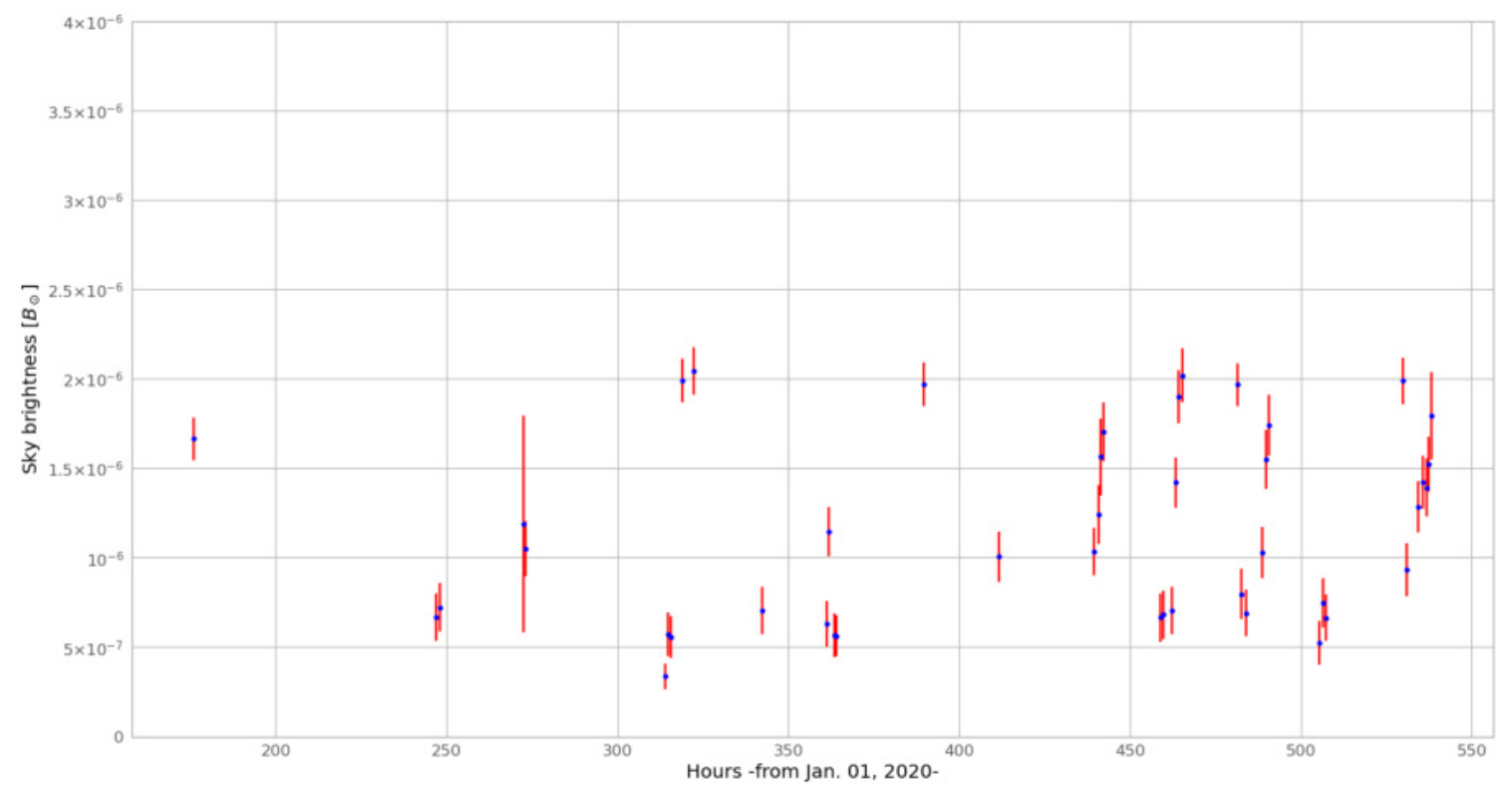

Figure 25. Sky brightness $\left[\mathrm{B}_{\odot}\right]$ measurements obtained during the XXXV Italian Antarctic Campaign (2019-2020), Concordia Base - Dome C, 3230 m a.s.l., Antarctica. On x-axis, the acquisition UTC time from January 1, 2020.

\section{ACKNOWLEDGMENTS}

This paper has been possible thanks to the whole ESCAPE Project team. The authors thank the OPTEC S.p.A for the AntarctiCor telescope thermal and structural design and realization and all persons who in any way contributed to the results reported in this manuscript. A particular acknowledgment is due to the Italian Piano Nazionale Ricerche Antartico (PNRA) ${ }^{7}$ thanks to which the ESCAPE project was able to take shape. Indeed, the AntarctiCor-ESCAPE project is funded by the PNRA, grant N. 2015-AC3.02. Additionally, the authors thank ALTEC Company for providing logistic support during the many AntarctiCor calibration periods and the European Space Agency (ESA) for its support to the PROBA-3/ASPIICS mission. 


\section{REFERENCES}

[1] Zecchino, M., "Polarization camera for image enhancement." 4D Technology Corporation - PolarCam User Manual, https://www.4dtechnology.com/products/polarimeters/polarcam/ (May 2017).

[2] Collett, E., [Polarized light. Fundamentals and applications], New York: Marcel Dekker (1992).

[3] MKS-Newport, "Resolution test targets." https://www.newport.com/f/resolution-test-targets?q= resolution\%20target:relevance\#features (2019).

[4] Sparks, W. and Axon, D., "Panoramic polarimetry data analysis," Publications of the Astronomical Society of the Pacific 111(764), 1298-1315 (1999).

[5] Vorobiev, D. V., Ninkov, Z., and Brock, N., "Astronomical polarimetry with the RIT polarization imaging camera," Publications of the Astronomical Society of the Pacific 130, 064501 (apr 2018).

[6] Arnaud, J., Faurobert, M., and Fossat, E., "Dome C: An exceptional site for solar observations .," Mem. S.A.It. 78, 105 (Jan. 2007).

[7] cfr. www.italiantartide.it (2021).

[8] Fineschi, S., Capobianco, G., Massone, G., Susino, R., Zangrilli, L., Bemporad, A., Liberatore, A., Landini, F., Romoli, M., Damé, L., Christille, J. M., Sandri, P., Marmonti, M., and Galy, C., "Antarcticor: Solar coronagraph in antarctica for the escape project," IL NUOVO CIMENTO 42 C (2018).

[9] Galy, C., Fineschi, S., Galano, D., Howard, R. A., Kintziger, C., Kirschner, V., Koutchmy, S., Lamy, P., Mazzoli, A., Melich, R., Mestreau-Garreau, A., Renotte, E., Servaye, J. S., Stockman, Y., Thizy, C., and Zhukov, A., "Design and modelisation of ASPIICS optics," in [Solar Physics and Space Weather Instrumentation VI], Fineschi, S. and Fennelly, J., eds., 9604, 71 - 82, International Society for Optics and Photonics, SPIE (2015).

[10] Lyot, B., "Étude de la couronne solaire en dehors des éclipses. Avec 16 figures dans le texte.," Zeitschrift für Astrophisik 5, 73 (Jan. 1932).

[11] Tordi, M., Bartolozzi, M., Fineschi, S., Capobianco, G., Massone, G., and Cesare, S., "Illumination system in visible light with variable solar-divergence for the solar orbiter METIS coronagraph," in [Solar Physics and Space Weather Instrumentation VI], Fineschi, S. and Fennelly, J., eds., 9604, 202 - 216, International Society for Optics and Photonics, SPIE (2015).

[12] cfr. https://www.altecspace.it/en/facilities/solar-simulation-chamber (2021).

[13] EdmundOptics, "White diffusing glass." www.edmundoptics.com/p/75mm-dia-white-diffusing-glass/ 3841/ (2019). 